\title{
Wiener Kreis: Identifikation
}

\author{
Philosophie betreiben bedeutet nichts anderes als: die Begriffe und Sätze \\ der Wissenschaft durch logische Analyse klären. \\ Rudolf Carnap, Die alte und die neue Logik (1930/31)
}

\subsection{Herausforderung: Verlust wissenschaftlicher Objektivität}

Im Gegensatz zu den anderen beiden Fallbeispielen (Husserl und Kritische Theorie) gibt es zwischen Alfred Seidel und dem Wiener Kreis weder eine biografische noch eine im engeren Sinne philosophietheoretische Verbindung. Seidel, der, wie im dritten Beispiel deutlich werden wird, als Vorläufer der Kritischen Theorie verstanden werden kann, nimmt keine direkten Bezüge zum Korpus, in dessen Tradition der Logische Positivismus steht. Meiner Interpretation nach subsumiert Seidel generell unter >Positivismus< das, was späterhin als die Maximen des Wiener Kreises bekannt wurde. >Positivismus< wiederum, verstanden als ahistorische, keiner potenziellen Revision bedürftige Wahrheit, ist für Seidel unakzeptabel. Er sei überzeugt, schreibt er, »dass alle Meinungen und Empfindungen, sogar die Ergebnisse der exakten Wissenschaft, einem räumlich und zeitlich begrenzten Kulturkreise angehören « (Seidel 1927: 122, Herv. MD). Dies wiederum lege den Schluss nahe, dass »die eigene Weltanschauung nur Ausdruck einer Zeitepoche ist, dass auch sie überholt werden wird, wie so manche Auffassung, die mit Emphase als letzte Wahrheit verkündet wurde« (ebd.).

Seidels Diagnose kann als Ausdruck eines allgemeineren Verständnisses gesehen werden, dass nämlich am Anfang des 20. Jahrhundert auch die exakten Wissenschaften Krisen erfahren. Bevor die Analysen zum Wiener Kreis beginnen, soll an einem Beispiel - an zwei Textausschnitten aus dem Band Krise und Neuaufbau in den exakten Wissenschaften (1933) - in knapper Form greifbar gemacht werden, was damit gemeint ist. Die Verfasser des Vorworts ${ }^{72}$ dieses Buches schreiben: »In Wahrheit sind die exakten Wissenschaften vor Krisen

72 Die Verfasser des Vorworts werden nicht angegeben. Der Band enthält fünf Vorträge (»Die Erschütterung der klassischen Physik durch das Experiment« von Hermann Mark, »Die Wandlung des Begriffssystems der Physik« von Hans Thirring, »Die Krise der Anschauung « von Hans Hahn, »Die vierte Dimension und der krumme Raum« von Georg Nöbeling sowie »Die neue Logik« von Karl Menger). 
keineswegs gesichert und gerade in den letzten Jahrzehnten sind sie von der theoretischen Physik bis hinein in die Logik von schweren Krisen erschüttert worden « (Deuticke 1933: o.S.). Was bisher als exakt angesehen worden sei, könne heute nur noch als durchschnittlich gelten, was als apriorisch gegolten habe, werde heute als empirischer Nachprüfung bedürftig erkannt. Wo früher nach Sicherem gefragt worden sei, frage man heute nach Wahrscheinlichem, und wo eine Erklärung gesucht wurde, suche man heute nach einer Beschreibung (ebd.). »Ja selbst in vielem, was man bewiesen zu haben glaubte, können wir nichts anderes mehr erblicken, als eine Transformation gewisser Ausgangssätze in gewisse andere Sätze, wobei sowohl die Ausgangssätze als auch die Transformationsregeln abänderbar sind « (ebd.). Kurz: Die hier exemplarisch zitierte Textstelle verdeutlicht eine Krise, nämlich dass die exakten Wissenschaften durch ihre eigene Ausdifferenzierung und Spezialisierung qua Anspruch auf Exaktheit nicht mehr exakt, apriorisch, sicher, erklärend und beweisend sind. Im Gegensatz zu Seidel, der durch Befunde dieser Art die wissenschaftliche Wahrheit und die Funktion der Wissenschaften allgemein infrage gestellt sah, verkünden die Verfasser des Vorworts von Krise und Neuaufbau in den exakten Wissenschaften - gleich nachdem sie die Symptome der Krise aufgelistet haben - optimistisch: »Aber ein Neuaufbau hat auf der ganzen Linie bereits erfolgreich begonnen!« (ebd.).

Die Verfeinerung unserer experimentellen Hilfsmittel hat zu außerordentlicher Reichweite und Genauigkeit unserer Beschreibungen geführt; Beobachtungen und Entdeckungen haben riesige neue Gebiete der Forschung entstehen lassen; ungeahnte Zusammenhänge wurden erkannt und führten zu neuen Begriffen und Begriffssystemen; die Argumentationen der Mathematik erreichten völlige logische Strenge; jenseits der Anschauung wurden rein deduktiv neue mathematische Systeme entwickelt, die sich vielfach physikalischer Anwendungen fähig erwiesen; und endlich wurden - zum ersten Male seit undenklichen Zeiten - wesentliche neue Erkenntnisse über das logische Schließen selbst gewonnen. (ebd.: o.S.)

Der Neuaufbau in den exakten Wissenschaften wird als unaufhaltsam beschrieben: »Es gibt kein Gebiet der exakten Wissenschaften, in dem die Bestrebungen eines Neuaufbaues nicht von Erfolg begleitet worden wären« (ebd.).

DieSelbstpositionierungsstrategien desWiener Kreises sindinnerhalbdieser »Linie«, die durch den wissenschaftlichen Fortschrittsgedanken geprägt ist, verortet. Seidel war dieser wissenschaftliche Optimismus fremd. Im Gegenteil drehen sich seine Fragen in Bewußtsein als Verhängnis immer wieder um das Problem, wie wissenschaftliche Erkenntnisse noch legitimierbar sind, wenn 
deren Objektivität grundlegend infrage gestellt wird. In letzter Konsequenz zeichnet er diesbezüglich ein Szenario der Unmöglichkeit. Die Problematik ist bereits in seinen Anfangssätzen angelegt, in denen er die allgemein feststellbare Glorifizierung und Verherrlichung des wissenschaftlichen Fortschritts entschieden als zu kurz gegriffen auffasst. »Alles glaubt man auf Grund ihrer Erkenntnisse [der Wissenschaft, MD] erreichen zu können. Daß dieses Wissen aber auch andere als die gepriesenen Wirkungen haben kann, das soll hier [in Seidels Buch, MD] gezeigt werden « (ebd.: 71). Seidel kritisiert - mit Husserl die »Wissenschaftsverfallenheit« seiner Zeit: »Wie kindlich optimistisch sind die Zeiten des Rationalismus in dem überschwänglichen Glauben an die Wirkungen des Erkennens; trotz aller Kritik ist noch heute das Pathos der Wissenschaft vielfach von diesem Glauben getragen « (ebd.: 75).

Im Unterschied jedoch zu Husserl, dessen Positionierungsstrategien u.a. auf die Legitimierung eines unabhängigen Status der Philosophie zielen und der die Haltung vertritt, dass wissenschaftliche Entscheidungen den »Stempel der Ewigkeit « besitzen (Husserl 1910/11: 337), macht Seidel klar, dass seine Untersuchungen nicht wertneutral sind. »Wertfreiheit bei charakterologisch gemeinten Untersuchungen wie der vorliegenden kann es nicht geben - noch viel weniger als bei den anderen Geisteswissenschaften « (Seidel 1924: 75). Ebenso unmöglich sei eine »metaphysikfreie Behandlung der Probleme« (ebd.). Zwar habe er die metaphysische Grundlage seiner Arbeit bewusst »herausgezogen « (da es sich bei der Untersuchung um eine psychologische und soziologische Fragestellung handele), sie mache sich aber »sofort bemerkbar, wo Erklärungen gegeben werden« (ebd.: 71-72). Metaphysische Voraussetzungen, so impliziert Seidel auf der einen Seite, durchzögen die Wissenschaften und die Philosophie zwingend. Auf der anderen Seite seien metaphysische Voraussetzungen nie »wertneutral«, d.h. nie objektiv. Das Seidel'sche Dilemma wird hier besonders deutlich: Von welchem Standpunkt aus können wissenschaftliche Erkenntnisse kritisch reflektiert werden, wenn diese umstandslos als gültig anerkannt werden? In seiner Fragestellung bringt Seidel das Dilemma auf den Punkt: »Wir stehen also mit den Verteidigern der Wissenschaften auf dem Standpunkt, daß die Welt des Sozialen und Psychischen - innerhalb gewisser Grenzen - erkennbar sei, und daß schon vieles erkannt wurde. Wir stellen demgegenüber die Frage, ob die Erkenntnis der sozialen und psychischen Vorgänge nicht gerade ihre Beeinflussung unmöglich macht, zumindest hemmt - die Frage nach dem Bewußtsein als Verhängnis« (ebd.: 74).

Weiter oben wurde argumentiert, dass in dieser Frage die Verluste philosophischer Standpunkte sichtbar werden: Seidel nimmt keine 
metawissenschaftliche philosophische Position ein, von der aus er eine Reflexion auf andere Wissenschaften vollziehen könnte. Als Folge davon verfängt er sich in einem erkenntnistheoretischen Zirkel, aus dem er keinen Ausweg weiß. An den Vertretern des Wiener Kreises kann eine Reaktion auf genau dieses Dilemma beobachtet werden: Sie aktualisieren in ihren Erneuerungsstrategien die Möglichkeit einer metawissenschaftlichen Position, der ein objektiver und universeller Status zukommt. Wie im Folgenden gezeigt wird, ist dafür zum einen eine Identifikation der Philosophie mit den exakten Wissenschaften, zum anderen die radikale Ausklammerung alles Metaphysischen aus dem Bereich des Wissenschaftlichen leitend.

Der Wiener Kreis gilt in der Philosophiegeschichte als Ursprung des Logischen Positivismus bzw. Logischen Empirismus ${ }^{73}$ und demnach als wichtiger Wegbereiter für die Entwicklung der Wissenschaftstheorie und der sogenannten analytischen Philosophie. ${ }^{74}$ Genau wie die Phänomenologie und die Kritische Theorie ist diese Tradition ein fixer Bestandteil des philosophischen Kanons des 2o. Jahrhunderts. Die analytische Philosophie gilt darüber hinaus als jene Tradition, die heute die philosophischen Fakultäten dominiert. Analog zu den anderen beiden Kapiteln werde ich im Folgenden im ersten Teil mein Untersuchungsmaterial vorstellen und daraus meine Hypothesen entwickeln (4.2). In einem zweiten Teil werde ich die Erneuerungsstrategien des Wiener Kreises an konkreten Textstellen nachzeichnen (4.3). In einem dritten Teil werde ich die bisherigen Ergebnisse meiner Arbeit in einem kurzen Zwischenfazit zusammenfassen (4.4). In einem letzten Teil schließlich, der gleichzeitig als Überleitung zum Kapitel über die Kritische Theorie fungiert, gehe ich auf die Gründe für die große Wirksamkeit der Erneuerungsstrategien des Wiener Kreises ein (4.5).

\subsection{Eine eigene Zeitschrift, Kongresse und eine Werbebroschüre}

Im Jahr 1895 wurde der Physiker Ernst Mach auf einen eigens für ihn eingerichteten Lehrstuhl der Philosophie an die Universität Wien berufen. Diese Berufung wird in der Philosophie- und Wissenschaftsgeschichte als wichtiges Ereignis wahrgenommen: »Dass ein Naturwissenschaftler philosophisch dilettierte, mochte noch angehen; doch dass er eine philosophische

73 Die Begriffe Logischer Positivismus und Logischer Empirismus werden hier synonym verwendet.

74 Ernest Nagel hat den Wiener Kreis bereits in seinen Impressions and Appraisals of Analytic Philosophy in Europe von 1936 als »analytische Philosophie« bezeichnet (Nagel 1936: 9). 
Lehrkanzel übernahm, ohne je über Kantianer oder Scholastiker examiniert worden zu sein, schien schon allerhand « (Sigmund 2015: 12). Mach und auch seinem Nachfolger Ludwig Boltzmann wird ein »disziplinüberschreitende[r] Einfluss « (Stadler 1993: 17) zugesprochen, der für die Entwicklung der Wissenschaftstheorie ausgehend vom Wiener Kreis entscheidend gewesen sei.

Naturwissenschaftler wie Mach, Fechner, Helmholtz oder Boltzmann treten in den philosophischen Diskurs ein, obwohl sie akademisch nicht dem Fachbereich Philosophie zugordnet sind beziehungsweise erst durch speziell eingerichtete Institutionalisierungsformen wie die Wiener Professur für »Philosophie, insbesondere Geschichte und Theorie der induktiven Wissenschaften « innerhalb der Philosophie institutionell sanktioniert werden. (Ziche 2008: 73)

Dieser institutionell verankerte disziplinäre Zusammenschluss zwischen Naturwissenschaften und Philosophie bot später für den Wiener Kreis die Gelegenheit, das breite Interesse an einer >wissenschaftlichen Philosophie< zu begründen: »Das Wirken der Physiker Mach und Boltzmann auf philosophischer Lehrkanzel läßt es begreiflich erscheinen, daß für die erkenntnistheoretischen und logischen Probleme, die mit den Grundlagen der Physik zusammenhängen, lebhaftes Interesse herrschte« (Neurath/Hahn/Carnap 1929: 302).

Die Traditionslinie von Mach bzw. Boltzmann zum Wiener Kreis, die bereits vom Wiener Kreis selbst angelegt worden ist, wird in der Philosophiegeschichtsschreibung bis heute fortgeschrieben: Die Geschichte des Wiener Kreises beginnt mit der Philosophie Machs. Innerhalb dieser Traditionslinie wiederum gilt der Wiener Kreis als entscheidender Wegbereiter für die Etablierung einer neuen, die Philosophie des 20. Jahrhunderts prägenden Subdisziplin der Philosophie: der Wissenschaftstheorie. »Tatsächlich aber etablierte sie [die Gruppe von Philosophen, Mathematikern, Physikern und Sozialwissenschaftlern, MD] innerhalb kurzer Zeit von zwanzig Jahren die Disziplin der Wissenschaftstheorie, die vordem als ein bloßer Annex der LogikLehrbücher in Erscheinung getreten war, als das bevorzugte Gebiet philosophischer Analyse. Damit führte sie die mit Mach begonnene Tradition zu einem sich weltweit auswirkenden Erfolg« (Haller 1993: 2-3). Als Stichdatum für die Entwicklung der Wissenschaftstheorie, die vom Wiener Kreis ausgeht, gilt das Jahr 1929. ${ }^{75} »$ Die internationalen Kongresse für Logik, Methodologie und Philosophie der Wissenschaften, die in unserer Zeit alle vier Jahre

75 Die Geschichte des Wiener Kreises wird üblicherweise wie folgt beschrieben: der »erste Wiener Kreis« (1907-1912), »Konstituierungsphase« (1918-1924), »nicht öffentliche Phase $«(1924-1928)$ und »öffentliche Phase« (1928-1934). 
stattfinden, sind die Fortsetzung der von den Wiener und Berliner logischen Empiristen veranstalteten Konferenzen, die 1929 ihren Anfang nahmen" (ebd.: 3). Dieses Datum fällt mit der »1. Internationalen Tagung für Erkenntnislehre der exakten Wissenschaften ${ }^{76}$ zusammen, die unter der Leitung des Mathematikers und Physikers Philipp Frank in Prag stattgefunden hat. Mit dieser Tagung hat sich der Wiener Kreis erstmals an eine breitere wissenschaftliche Öffentlichkeit gewandt. ${ }^{77}$ Dieser erste >öffentliche Auftritt< des Wiener Kreises wird in zwei Publikationen manifest. Zum einen erscheint 1930/31 der erste Band der neu lancierten Zeitschrift »Erkenntnis«, herausgegeben von Rudolf Carnap aus Wien und Hans Reichenbach aus Berlin, zum anderen 1929 die Broschüre Wissenschaftliche Weltauffassung. Der Wiener Kreis. Beide Publikationen dienen als Grundlagen für die Analysen dieses Kapitels und sollen kurz vorgestellt werden.

\section{Die erste Ausgabe der »Erkenntnis«}

Die »Erkenntnis« ging aus der Zeitschrift »Annalen der Philosophie« hervor, die seit 1919 im Verlag von Felix Meiner erschien. Im Zusammenhang mit der »1. Internationalen Tagung für Erkenntnislehre der exakten Wissenschaften « lernte Felix Meiner einige Mitglieder des Wiener Kreises und der Berliner Gruppe kennen. ${ }^{78}{ } \gg$ Dabei muss auch das starke Interesse von Reichenbach und Carnap an einer redaktionellen Übernahme der in Schwierigkeiten steckenden Zeitschrift deutlich geworden sein, während andererseits Felix Meiner offenbar den Eindruck gewann, durch enge Anbindung der Zeitschrift an die Bewegung des Logischen Empirismus die Zeitschrift sinnvoll weiterführen zu können « (Hegselmann/Siegwart 1991: 463). Reichenbach als Vertreter der Berliner Gruppe und Carnap als Vertreter des Wiener Kreises übernahmen als Herausgeber die »Annalen«, führten sie aber als »Erkenntnis« weiter. Träger der Zeitschrift waren die »Gesellschaft für empirische Philosophie« aus Berlin

76 Es handelte sich dabei um die »Jahrestagung der Deutschen Physiker und Mathematiker«. Weitere Tagungen und Kongresse: 1934 - Prager Vorkonferenz des Internationalen Kongresses für Einheit der Wissenschaft; 1935 - Paris, Erster Internationaler Kongress für Einheit der Wissenschaft; 1936 - Kopenhagen, Zweiter Internationaler Kongress für Einheit der Wissenschaft; 1937 - Paris, Dritter Internationaler Kongress für Einheit der Wissenschaft; 1938 - Cambridge, Vierter Internationaler Kongress für Einheit der Wissenschaft: die wissenschaftliche Sprache; 1939 - Cambridge Mass., Fifth International Congress for the Unity of Science; 1941 - Chicago, Sixth International Congress for the Unity of Science (Stadler 1997: 427-436).

77 Bereits ein Jahr vorher, 1928, erschien das erste Buch einer vom Wiener Kreis zwischen 1928-1937 herausgegebenen Buchreihe: Schriften zur wissenschaftlichen Weltauffassung, hrsg. von Philipp Frank und Moritz Schlick.

78 Das Verhältnis der beiden Gruppen wird weiter unten thematisiert. 
(als das öffentliche Forum der Berliner Gruppe) und der »Verein Ernst Mach « aus Wien (als das öffentliche Forum des Wiener Kreises). Obwohl Reichenbach und Carnap beide als offizielle Herausgeber fungierten, soll Reichenbach an der Edition weitaus mehr beteiligt gewesen sein als Carnap ${ }^{79}$ (Milkòv 2011: XVII). Reichenbach ist es auch, der in der ersten Ausgabe den Beitrag »Zur Einführung « verfasst, in dem das neue Programm der »Erkenntnis « vorgestellt wird. Reichenbach stellt einen Bezug zur Vorgängerzeitschrift, zu den »Annalen der Philosophie « her: »War es schon immer das Programm der >Annalen $<$ gewesen, Philosophie nicht als isolierte Wissenschaft, sondern im engsten Zusammenhang mit den einzelnen Fachwissenschaften zu treiben, so darf der Wechsel in der Schriftleitung als eine Unterstreichung dieses Standpunktes betrachtet werden « (Reichenbach 1930/31: 1). Werden jedoch die Programme der beiden Zeitschriften miteinander verglichen, wird ein entscheidender Unterschied deutlich. Hans Vaihinger und Raymund Schmidt, die Herausgeber der »Annalen«, betonen 1919 in ihrer ersten Ausgabe die Wichtigkeit einer Zusammenarbeit zwischen Philosophie und empirischen Fachwissenschaften:»Bisher hat es an einer fruchtbaren Wechselwirkung zwischen Einzelwissenschaften und Philosophie gefehlt. Wohl haben gelegentlich und mehr zufällig solche gegenseitigen Förderungen stattgefunden, aber es fehlte an einem gemeinsamen Platze, auf dem Philosophen einerseits und Vertreter der einzelnen Fachwissenschaften andererseits zusammen kommen und zusammen arbeiten konnten « (Vaihinger/Schmidt 1919: V). Im Unterschied zu den Herausgebern der »Annalen «, die eine Zusammenarbeit zwischen Philosophen und Einzelwissenschaftlern angestrebt haben, stellt Reichenbach in seiner Ankündigung - wenn auch etwas vorsichtig - als Ziel die Auflösung der Fächergrenzen in den Vordergrund. »Es ergibt sich [...] aus unserer Zielsetzung, daß der Schwerpunkt der Zeitschrift in den Arbeiten liegt, die ihre Quellen in dem ertragreichen Boden der Empirie haben; der Strom philosophischer Erkenntnisse, der in unserer Zeit reicher als je aus den Einzelwissenschaften fließt, soll in unserer Zeitschrift ein Bett finden, das ihn fasst und weiterführt und ihm geordnete Bahnen weist « (Reichenbach 1930/31: 1). Philosophie, so die Implikation, bestehe nicht aus einer Einzeldisziplin, sondern aus einer Tätigkeit, die sich in den Fachwissenschaften aufzulösen habe. »Die jetzigen Herausgeber haben es von jeher als Aufgabe betrachtet, Philosophie im Sinne von Wissenschaftskritik zu treiben und durch wissenschaftsanalytische Methoden diejenigen Einsichten in Sinn und Bedeutung menschlicher Erkenntnis zu gewinnen, welche die in immer neuen Systemen

79 Die ungleiche Rollenverteilung der beiden Herausgeber widerspiegelt sich in den ungleich groß gedruckten Namen auf den Deckblättern der ersten vier Ausgaben. 
formulierte, auf ein angenommenes Eigenrecht der Vernunft gegründete Philosophie der historischen Schulen vergeblich gesucht hatte« (ebd.). Wissenschaftsanalytische Methoden, so Reichenbach, bärgen das Potenzial, die >alte< und überholte Philosophie zu ersetzen. »Es ist unsere Überzeugung, daß [...] die Philosophie als Wissenschaft eine neue Grundlegung erfahren wird « ${ }^{80}$ (ebd.: 2). Während Vaihinger und Schmidt die »Annalen« als Forum lanciert haben, in dem Einzelwissenschaftler und Philosophen zusammenkommen können, präsentiert Reichenbach die »Erkenntnis« als Forum, in dem Einzelwissenschaftler philosophische Erkenntnisse vorstellen sollen. Dadurch wiederum, so Reichenbach, erfahre die Philosophie eine neue Grundlegung. Die Verschiebung in der Programmatik, wie sie in den beiden Vorwörtern der Zeitschriften augenscheinlich wird, verdeutlicht exemplarisch, worin der Erneuerungsanspruch des Wiener Kreises besteht: Durch ihre Rückbeziehung auf die Einzelwissenschaften kann sich die Philosophie neu fundieren. Umgekehrt bedeutet dies auch, dass die analytisch informierte Wissenschaftsphilosophie die >alte< und überholte Philosophie ersetzen soll. ${ }^{81}$

ImerstenBand der»Erkenntnis «sind Reden und Aufsätzepubliziert, indenen dieser Erneuerungsanspruch - wenn auch in verschiedenen Nuancierungen programmatisch vertreten wird. Insbesondere Schlicks »Die Wende der Philosophie « und Carnaps »Die alte und die neue Logik« sind kanonisch geworden und werden heute als Standardaufsätze des Wiener Kreises behandelt. Ein weiterer berühmter Aufsatz wurde im Folgeband von 1931 erstmals veröffentlicht: Carnaps »Überwindung der Metaphysik durch logische Analyse der Sprache «. In der »Erkenntnis« sind zudem die Kongresse des Wiener Kreises dokumentiert. Es seien diese Kongressdokumentationen gewesen - so betonen Rainer Hegselmann und Geo Siegwart -, die dafür verantwortlich waren, dass die »Erkenntnis« während ihrer Laufzeit von 1930 bis 1939 die Rolle des Zentralorgans des Logischen Empirismus eingenommen hat (Hegselmann/ Siegwart 1991: 464). Kurz: Es handelt sich bei der »Erkenntnis« um einen geglückten Versuch einer Neulancierung. An den hier versammelten Texten

8o Eine solche direkte Aussage über die Erneuerung der Philosophie, wie sie Reichenbach trifft, ist in den untersuchten Texten selten. Üblicherweise wird nicht die Erneuerung der Philosophie, sondern ihre Verabschiedung betont, da unter einer logischen bzw. sprachanalytischen Perspektive beinahe jegliche bisherige Philosophie den Stempel der Metaphysik erhält. Die Verwendung des Philosophiebegriffs wird, wie weiter unten deutlich wird, von Reichenbach und Carnap bzw. vom Wiener Kreis unterschiedlich beurteilt.

81 »Denn mit dem expliziten Auftreten der Wissenschaftstheorie als eigener systematischer Disziplin verband sich von allem Anfang an auch die Idee einer totalen Ersetzung der alten Philosophie durch eine neue. So erklärt Carnap ganz ausdrücklich [in Logische Syntax der Sprache, MD]: >An die Stelle des unentwirrbaren Problemgemenges, das man Philosophie nennt, tritt die Wissenschaftslogik« (Oeser 2003: 93). 
wird die Formierung eines bestimmten »Denkstils« besonders deutlich (Fleck $1980[1935]) .^{82}$

\section{Ein Manifest}

1929 erschien unter der Herausgeberschaft des »Verein Ernst Mach« die Broschüre Wissenschaftliche Weltauffassung. Der Wiener Kreis. Das Werk habe »keine offiziell genannten Verfasser« und sei »Moritz Schlick gewidmet« (Neurath/Hahn/Carnap 1929: 299). Als Verfasser gelten Hans Hahn, Rudolf Carnap und Otto Neurath; die Studierenden Friedrich Waismann und Herbert Feigl sollen ebenso dazu beigetragen haben wie Maria Kaspar (Sigmund 2015: 127). Die Programmatik des Textes ist signifikant:

Die wissenschaftliche Weltauffassung ist nicht so sehr durch eigene Thesen charakterisiert als vielmehr durch die grundsätzliche Einstellung, die Gesichtspunkte, die Forschungsrichtung. Als Ziel schwebt die Einheitswissenschaft vor. Das Bestreben geht dahin, die Leistungen der einzelnen Forscher auf den verschiedenen Wissenschaftsgebieten in Verbindung und Einklang miteinander zu bringen. Aus dieser Zielsetzung ergibt sich die Betonung der Kollektivarbeit [...]. Sauberkeit und Klarheit werden angestrebt, dunkle Fernen und unergründliche Tiefen abgelehnt. In der Wissenschaft gibt es keine »Tiefen«; überall ist Oberfläche [...]. Alles ist dem Menschen zugänglich; und der Mensch ist das Maß aller Dinge. [...] In solcher Weise wird durch die logische Analyse nicht nur die Metaphysik im eigentlichen, klassischen Sinne des Wortes überwunden, insbesondere die scholastische Metaphysik und die der Systeme des deutschen Idealismus, sondern auch die versteckte Metaphysik des Kantischen und des modernen Apriorismus. Die wissenschaftliche Weltauffassung kennt keine unbedingt gültige Erkenntnis aus reiner Vernunft, keine "synthetischen Urteile a priori«, wie sie der Kantischen Erkenntnistheorie und erst recht aller vor- und nachkantischen Ontologie und Metaphysik zugrunde liegen [...]. Die wissenschaftliche Weltauffassung kennt nur Erfahrungssätze über Gegenstände aller Art und die analytischen Sätze der Logik und Mathematik. (Neurath/Hahn/ Carnap 1929: 305-307)

Der Wiener Kreis tritt mit dem Anspruch auf, die abendländische Metaphysik mit den Mitteln der logischen Analyse ein für alle Mal überwinden zu können. Gemäß Karl Mengers Erinnerungen ist diese Schrift entstanden, weil Schlick die damalige Zentralfigur des Wiener Kreises - sich entschieden hatte, einen Ruf nach Bonn abzulehnen, um in Wien zu bleiben:

82 Der Bezug auf Flecks Konzept des Denkstils bzw. Denkkollektivs vermag hier der Schwierigkeit zu begegnen, dass es sich beim Wiener Kreis um eine Ansammlung unterschiedlicher Autoren handelt. Den Wiener Kreis als eigentliches >Agens` gibt es nicht. Formulierungen, die im Folgenden in diese Richtung zielen, sind mit Rückbezug auf das Konzept von Fleck zu verstehen. 
Groß war die Freude aller von uns, als wir erfuhren, dass Schlick sich entschieden hatte, weiter in Wien zu bleiben. »Das muss gefeiert werde[n] «, sagte Neurath, und wir stimmten zu. »Wir müssen ein Buch verfassen, das unsere Ansichten darstellt - ein Manifest des Kreises - und es Schlick zu seiner Rückkehr im Herbst widmen«, fügte Neurath hinzu. Und mit der ihm üblichen Geschäftigkeit und Energie ging er ans Werk. (Karl Menger, zitiert nach Sigmund 2015:126)

Das »Manifest « war sowohl innerhalb als auch außerhalb des Wiener Kreises umstritten und hat damals enervierte Reaktionen ausgelöst. Carnap bereitet Schlick in einem Brief umsichtig auf die Lektüre vor: »Ich werde dir jetzt dein Exemplar übersenden, an dem du dein blaues Wunder erleben wirst; hoffentlich nur äußerlich. Den Inhalt betrachte bitte nicht zu kritisch, sondern mit deinem gewohnten Wohlwollen und Nachsicht. Es ist von Feigl, Neurath und mir mit vereinten Kräften und mehr gutem Willen als Qualität geschaffen worden« (Carnap an Schlick, 30.o9.1929, zitiert nach Sigmund 2015: 128). Carnap habe wohl geahnt, so der Mathematiker Karl Sigmund, der sich mit der Geschichte des Wiener Kreises auseinandergesetzt hat, »dass Schlick sich an einigen ziemlich reklamehaften Passagen und dogmatischen Äußerungen stoßen könnte [...] (Sigmund 2005: 129). Menger wiederum habe aus der Schrift Konsequenzen gezogen: Er wollte nach der Lektüre nicht mehr als Mitglied des Wiener Kreises, sondern nur noch als ein dem Kreis Nahestehender gelten (ebd.). Am heftigsten soll jedoch Ludwig Wittgenstein reagiert haben. ${ }^{83}$ An Friedrich Waismann schrieb er: »Das ist eine schwere Geschichte. Und es ist mir sehr unangenehm zu denken, dass hier wieder einmal ein an sich guter Grund als Anlass zu G'schaftlhuberei benutzt werden soll« (Wittgenstein an Waismann, Juli 1929, zitiert nach Sigmund 2015: 129). Schlick verdiene es,

dass man sich davor hütet, ihn und die Wiener Schule, deren Exponent er ist, »in guter Absicht« durch Großsprecherei lächerlich zu machen. Wenn ich sage Großsprecherei, so mein ich damit jede Art der Selbstbespiegelung. »Absage an die Metaphysik«! Als ob das was Neues wäre! - Das Lob, das eine Schule sich selbst spendet, stinkt wie jedes Eigenlob. (ebd.)

83 Die Beziehung zwischen Wittgenstein und dem Wiener Kreis wird von Schnädelbach wie folgt beschrieben: »Der >Tractatus wird vom Logischen Empirismus des Wiener Kreises gewissermaßen okkupiert und zu seiner Gründungsurkunde erklärt « (Schnädelbach 1992: 310). Zum Verhältnis Wittgenstein - Wiener Kreis vgl. z.B. Stadler 1997: 467-488. »Aus wissenschaftssoziologischer Sicht kann man - im Anschluss an die Arbeiten von Ludwik Fleck und Thomas S. Kuhn - vom Aufeinanderprallen zweier Denkstile beziehungsweise eines Denkkollektivs und eines Individualisten sprechen; ein Gegensatz, der aber von einer durchaus bewusst partiellen Rezeption Wittgensteins durch einen Teil des Wiener Kreises mitbestimmt ist« (ebd.: 481). 
Das »Manifest «, so wird erkennbar, ist eine Schrift, deren Polemik von einigen Mitgliedern des Wiener Kreises und auch von Wittgenstein abgelehnt worden ist. Als eine Art Werbebroschüre spielte sie im Zusammenhang mit der genannten Tagung und der Lancierung einer eigenen Schriftenreihe im Übergang von der >privaten $<$ zur >öffentlichen< Phase des Wiener Kreises jedoch eine wichtige Rolle. »Eigene Zeitschrift, Tagung, Werbebroschüre, vor allem aber ein publikumswirksamer Name - alles trug dazu bei, um den Schlick-Zirkel (in Wien hieß er naturgemäß weiter so) von der privaten Phase zur öffentlichen überzuleiten« (Sigmund 2005: 128). Das »Manifest« verdeutlicht exemplarisch, dass die »wissenschaftliche Weltauffassung « - wie Donata Romizi schreibt als eine Botschaft für die Gesellschaft gemeint war (Romizi 2012:146).

\section{Die Berliner Gruppe und der Wiener Kreis}

Nikolay Milkòv, der sich in jüngerer Zeit um eine Wiederveröffentlichung und Neubewertung von Reichenbachs Schriften bemüht hat, betont, dass die Veröffentlichung des »Manifests« »die nachfolgende Wahrnehmung der Bewegung, die heute als logischer Empirismus verstanden wird, entscheidend beeinflusst [hat] « (Milkòv 2015: XXI). Vor der Veröffentlichung sei nämlich Reichenbach - und nicht Carnap oder Schlick - in Deutschland der Hoffnungsträger für die Etablierung einer wissenschaftlichen Philosophie gewesen (ebd.). Milkòvs Bemerkung verweist erstens auf die grundsätzliche Tatsache, dass nicht etwa lediglich der Wiener Kreis für die Entwicklung der Wissenschaftstheorie verantwortlich ist, sondern sich eine große Anzahl von Wissenschaftlern und Wissenschaftlerinnen aus unterschiedlichsten Disziplinen mit dem Thema der Verbindung von Naturwissenschaften und Philosophie auseinandergesetzt hat. Die Bemerkung verweist zweitens auf die zentrale Rolle Reichenbachs für den Wiener Kreis. Zwar haben - wie erwähnt - offiziell Carnap und Reichenbach gemeinsam die Herausgeberschaft der »Erkenntnis« übernommen, die Zeitschrift wurde jedoch von Berlin aus geleitet, d.h., Reichenbach war inoffizieller Hauptherausgeber. 1938 schrieb Reichenbach in einem Brief: »I may add here the remark that >Erkenntnis〈 was not a foundation of the Vienna group, but of the Berlin group, and that I invited the Vienna group to collaborate in the edition of this journal Schlick refused to accept because of the differences in his views and mine « (Reichenbach an Max Black, 18.04.1938, zitiert nach Milkòv 2015: XII).

Reichenbach war die zentrale Figur der sogenannten Berliner Gruppe. Diese Gruppe umfasste u.a. Reichenbach, Walter Dubislav, Kurt Grelling, Kurt Lewin und gilt gemeinsam mit dem Wiener Kreis als entscheidende Vorstufe für die Entstehung des Logischen Empirismus. Die Unterschiede zwischen der 
Berliner Gruppe und dem Wiener Kreis ${ }^{84}$ verweisen auf einen Zusammenhang, der für die Argumentation des vorliegenden Kapitels zentral ist: Die Berliner Gruppe hielt - im Gegensatz zum Wiener Kreis - am Begriff der Philosophie fest. ${ }^{85}$ So habe Reichenbach für die »1. Tagung für Erkenntnislehre der exakten Wissenschaften « einen Titel vorgeschlagen, der von Carnap und Schlick aufgrund der Begriffsverwendung »Philosophie« abgelehnt worden ist: »Kongress für Naturphilosophie, oder naturphilosophischer Kongress « (zitiert nach Milkòv 2015:XVIII). Für Reichenbach kulminierte der Zusammenschluss der exakten Wissenschaften mit der Philosophie im Begriff der Naturphilosophie. Seinen Aufsatz »Ziele und Wege der heutigen Naturphilosophie«, der gemäß Milkòv als Programmschrift der »Gesellschaft für empirische Philosophie « verstanden werden sollte (Milkòv 2011: XXXV), beginnt Reichenbach mit folgenden Worten:

\begin{abstract}
Der Name Naturphilosophie gewinnt in unseren Tagen einen neuen Klang. Herausgewachsen aus den Resultaten naturwissenschaftlicher Forschung, beginnt eine neue philosophische Wissenschaft, erfüllt von Intensität und Strenge, ihr Dasein zu gestalten; sie hat bereits eine Reihe von alten Problemen zu ungeahnter Wendung geführt und ebenso eine Anzahl neuartiger Probleme aufgewiesen, deren Existenz man früher übersehen hatte. Es ist diese Naturphilosophie, deren Problemlage in dem folgenden Umriss dargestellt werden soll. (Reichenbach 2011 [1931]: 47)
\end{abstract}

Für den Wiener Kreis hingegen galt es, den Begriff der Naturphilosophie wie den der Philosophie überhaupt zu vermeiden. Neurath kommentiert Reichenbachs Aufsatz entsprechend mit folgenden Worten: » Naturphilosophie< ist an sich ein irreführender Ausdruck, weil er so klingt, als ob es neben wissenschaftlichen Sätzen noch sinnvolle philosophische [Sätze] geben könne «(Neurath an Meiner, ohne Datum, zitiert nach Milkòv 2015:XVIII). Zuerst soll Reichenbach empört reagiert und mit der Auflösung der Zeitschrift gedroht haben. In einem Brief an Carnap schreibt er schließlich: »Ich habe zu den Veröffentlichungen des Wiener Kreises bisher geschwiegen, obwohl manches schwerwiegende Fehler zu enthalten scheint « (Brief von Reichenbach an Carnap, 22.08.1931, zitiert nach Milkòv 2015: XVIII-XIX). Er plädierte für »gegenseitige Duldung«

\footnotetext{
$84 \quad$ Zur Berliner Gruppe und zum Unterschied zwischen der Berliner Gruppe und dem Wiener Kreis vgl. Milkov 2015. Unter dem Einfluss von Hans Reichenbach wurde die Berliner Gesellschaft 1931 in »Gesellschaft für wissenschaftliche Philosophie« umbenannt.

85 Die Ablehnung des Philosophiebegriffs bzw. dessen radikale Umbenennung ist eine wirkungsvolle Argumentationsstrategie und wird weiter unten wieder aufgenommen (siehe Kapitel »Philosophische Altlasten aus dem Weg räumen: Metphysikkritik«, 10o105, und Fazit, S. 193-194).
} 
(ebd.), nicht für mehr. Der Bund zwischen dem Wiener Kreis und der Berliner Gruppe war - so resümiert Milkòv - »offensichtlich nichts anderes als eine Zweckehe« (Milkòv 2015:XIX).

Vermutlich spielte diese »Zweckehe« für die öffentliche Wirkung des Wiener Kreises eine nicht unerhebliche Rolle. Die Programmatik des Wiener Kreises die Ersetzung der bisherigen Philosophie durch die Wissenschaftstheorie wurde dadurch sowohl durch internationale Vernetzung als auch durch andere wissenschaftstheoretische Ansätze legitimatorisch abgestützt. Denn trotz ihrer Unterschiede werden in den beiden Gruppierungen die gleichen Erneuerungsstrategien sichtbar: die Identifikation der Philosophie mit den exakten Wissenschaften auf der einen Seite und die Abgrenzung von der Metaphysik, d.h. der traditionellen Philosophie, auf der anderen Seite.

\section{Identifikation mit den Naturwissenschaften, Abgrenzung von der Philosophie}

Die Nähe zu den exakten Wissenschaften widerspiegelt sich bereits in den Promotionsfächern der Kernmitglieder des Wiener Kreises. ${ }^{86}$ So wurden Gustav Bergmann, Philipp Frank, Kurt Gödel, Hans Hahn, Olga Hahn-Neurath, Karl Menger, Richard von Mises, Marcel Natkin und Theodor Radaković in Mathematik oder Physik promoviert, Moritz Schlick, Rudolf Carnap, Herbert Feigl und Victor Kraft hingegen erhielten ihrer Doktorgrad in Philosophie. Otto Neurath wiederum hat in Nationalökonomie promoviert. ${ }^{87}$ Diese disziplinäre Zusammensetzung ist bezeichnend für die Erneuerungsstrategien des Wiener Kreises: Im Unterschied zu Husserl und zur Kritischen Theorie wird der Erneuerungsanspruch der Philosophie hier wesentlich aus einem

86 Diese Liste repräsentiert die Mitglieder, wie sie im »Manifest« als Kernmitglieder aufgelistet werden. Zum engeren Kern gehörten weiter Béla Juhos, Felix Kaufmann, Rose Rand, Josef Schächter, Edgar Zilsel.

87 Aufgelistet ist zusätzlich Friedrich Waismann. Er hat Mathematik, Physik und Philosophie studiert, aber nie eine Doktorarbeit eingereicht. Bekanntheit erlangt hat er u.a. durch die sogenannten Waismann-Protokolle: Zwischen 1927 und 1936 führte Waismann mehrere Gespräche mit Wittgenstein, die 1967 posthum unter dem Titel Ludwig Wittgenstein und der Wiener Kreis veröffentlicht wurden. Wittgenstein soll ein anspruchsvoller Gesprächspartner gewesen sein: »Jedes Mal, wenn er [Wittgenstein, MD] Waismanns Manuskript zu Gesicht bekam, verlangte er drastische Änderungen, und gelegentlich wieder Rückänderungen, oder einen komplett anderen Aufbau. [...] Wittgenstein war kaum je mit Waismanns Darstellung einverstanden, selbst wenn ihm dieser penibel nachweisen konnte, dass es sich um eine wortgetreue Niederschrift früherer Äußerungen handelte (Sigmund 2015: 229). Wittgenstein und Waismann hatten darüber hinaus den Plan, gemeinsam ein Buch zu verfassen. Das Projekt scheiterte schließlich an den philosophischen Differenzen von Wittgenstein und Waismann. 2003 wurden die Texte des Projekts veröffentlicht (Baker 2003). 
interdisziplinären Zusammenschluss heraus entwickelt und begründet. ${ }^{88}$ So handelte es sich bei der genannten Initialtagung in Prag um die Jahrestagung der »Deutschen Physiker und Mathematiker«. Philipp Frank, damals Physikprofessor in Prag und Organisator der Tagung, erinnert sich:

The arrangement of the meeting was not so easy. The ordinary regular philosophy meetings followed the traditional lines and would hardly have given us enough scope. By a happy coincidence I was just in 1929 arranging a meeting of the physicists and mathematicians from the German-speaking regions in Central Europe. The meeting was to be held in my place of residence, Prague, the capital of Czechoslovakia. The German Physical Society, which was the official sponsor of this meeting, did not particularly like the idea of combining this serious scientific meeting with such a foolish thing as philosophy. However, I was the chairman of the local committee in Prague and they could not refuse my serious wish to attack a meeting with the topic, »Epistemology of the Exact Sciences«. (Frank 1949: 39-40)

Franks Schilderung zeigt, dass sich die Vertreter des Wiener Kreises von den bestehenden philosophischen Kontexten distanzierten, um ihr Anliegen einer >reinen< wissenschaftlichen Philosophie im Kontext der Mathematik und Physik zu positionieren. Offensichtlich barg diese Einbringung philosophischer Themen in den Kontext der exakten Wissenschaften ein provokatives Potenzial.

There is no doubt that quite a few people in the audience were shocked by my blunt statements that modern science is incompatible with the traditional system of philosophy. Probably, most of the scientists had not been accustomed to thinking of philosophy and science as one coherent system of thought. Philosophy had been for them what the Sunday sermon is for a businessman who is only interested in profit. Philosophy had been required not to be »true « but to give emotional satisfaction. (ebd.: 41)

Frank erinnert nicht nur an die für den Wiener Kreis charakteristische Überschreitung der Disziplinengrenzen, sondern er verweist zugleich auf die zentrale - und im Folgenden weiter auszuführende - Argumentationsstrategie, nämlich genau dadurch eine philosophische Position sui generis zu restabilisieren. Die genuin interdisziplinär angelegte Situation im

88 Freilich werden auch die Erneuerungsansprüche der Kritischen Theorie mit dem Argument der Interdisziplinarität vorangetrieben. Wie jedoch im dritten Kapitel deutlich wird, entwickeln Horkheimer und Adorno ihre Erneuerungsansprüche nicht ausgehend von den zeitgenössischen soziologischen Diskussionen, sondern sie fordern eine Kooperation der Philosophie mit ebendiesen. Genau darin liegt ein wesentlicher Unterschied zwischen der Kooperationsstrategie (wie sie idealtypisch an der Kritischen Theorie deutlich wird) und der Identifikationsstrategie (wie sie idealtypisch am Wiener Kreis deutlich wird). 
Erneuerungsanspruch des Wiener Kreises verweist somit auf ein Modell der Identifikation: Durch die Identifikation ihrer eigenen philosophischen Position mit Methoden und Modellen aus der Physik und Mathematik, so die argumentative Stoßrichtung dieses Kapitels, stabilisieren und rehabilitieren die Vertreter des Wiener Kreises den Wahrheits- und Objektivitätsanspruch nicht nur der Philosophie, sondern der Wissenschaft im Allgemeinen.

Dass sich die Vertreter des Wiener Kreises in expliziter Weise von der traditionellen Philosophie distanzierten, indem sie sich nicht als Philosophen identifizierten, ist ein zentraler Bestandteil dieser Strategie. Ein Beispiel sei kurz vorgestellt: Jonas Cohn, ein Freiburger Neukantianer, soll 1935 bei Reichenbach angefragt haben, ob die Zeitschrift »Erkenntnis« bereit sei, eine Kritik des Wiener Kreises zu veröffentlichen. Carnap schreibt diesbezüglich in einem Brief an Neurath: »Reichenbach findet es nicht so schlimm und meint, die Einwände seien typisch und daher lohne es sich, sie zu bringen. Ich finde das Ganze viel zu philosophisch und für unsere Leser nicht aufklärend. Ich möchte lieber ablehnen. Was meinst du? Sollen wir Wert darauf legen, mit Philosophen zu diskutieren?« (Carnap an Neurath, 23.01.1935, zitiert nach Hegselmann/Siegwart 1991: 464). In Carnaps Bemerkung wird eine klare Distanzierung zu den »Philosophen« deutlich. Wo Carnap eine Diskussion mit den Philosophen jedoch noch in Erwägung zieht, betrachtet Neurath ein solches Unterfangen schlicht als Zeitverschwendung: »Ad Jonas Coh. Ich hälte [sic!] es für abwegig, in der Erkenntnis Auseinandersetzungen mit der traditionellen Philosophie zu bringen. Man hat kaum Raum für die internen Sachen ... Wir haben gar kein Interesse daran, uns mit stypischen Einwänden auseinanderzusetzen « (Neurath an Carnap, 28.01.1935, zitiert nach Hegselmann/Siegwart 1991: 464). Neurath beendet die Debatte, indem er die Absetzung von den Philosophen affirmiert: Dringlicher als die Diskussion mit den Philosophen seien die eigenen Anliegen.

Im Folgenden wird an konkreten Textstellen nachgezeichnet, wie diese Erneuerungsansprüche entwickelt werden. Da hier das Ziel verfolgt wird, argumentative Strategien zu erfassen, mit denen sich der Wiener Kreis im Kontext anderer Disziplinen legitimiert, wird nicht auf die inhaltlichen Differenzen bzw. auf die inhaltlich und methodologisch zuweilen stark divergierenden Positionen eingegangen. Das Ziel ist das Herausarbeiten eines bestimmten Denkstils. In einem ersten Schritt werde ich Auszüge aus Schlicks programmatischem Aufsatz »Die Wende der Philosophie« hinsichtlich seines Erneuerungsanspruchs analysieren. Mit diesem ersten Aufsatz - »einer von Wittgenstein inspirierten, euphorischen Bestandsaufnahme des >linguistic turn « (Stadler 1993: 24) - wird die »Erkenntnis« als die neue Plattform der wissenschaftlichen Philosophie vorgestellt (ebd.). Auf Schlicks Aufsatz folgt 
in der ersten Ausgabe der »Erkenntnis« Carnaps Beitrag »Die alte und die neue Logik«. An diesem Aufsatz zeichne ich in einem zweiten Schritt nach, wie der Wiener Kreis die Identifikation seiner philosophischen Position mit den Naturwissenschaften herleitet, nämlich durch eine Transponierung neuer Befunde aus der Mathematik in den Bereich der Philosophie. In einem dritten Schritt werde ich an Carnaps »Überwindung der Metaphysik durch logische Analyse der Sprache« darstellen, wie der Wiener Kreis seine eigene Position von der philosophischen Tradition absetzt. Schließlich wird gezeigt, dass der Wiener Kreis seinen Erneuerungsanspruch der Philosophie dadurch begründet, dass er die Philosophie als eine Methode, d.h. als logische Analyse, definiert. Mit dieser Bestimmung der Philosophie, die mit der Forderung nach einer Auflösung der Philosophie als einer eigenen Fachwissenschaft einhergeht, begründet der Wiener Kreis nicht nur seinen Anspruch, die Philosophie in jeder anderen Wissenschaft anwenden zu können, sondern er rehabilitiert darüber hinaus die philosophische Tätigkeit als - in Schlicks Worten - »Alpha und Omega aller wissenschaftlicher Erkenntnis«(Schlick 193o/31: 8).

\subsection{Philosophie als logische Analyse}

\section{Eine grundsätzliche Wende der Philosophie}

Die Feststellung, dass die Philosophie gegenwärtig eine totale und endgültige Wende erfahre, ist in den untersuchten Texten ein wiederkehrendes Moment. Schlicks Aufsatz »Die Wende der Philosophie« ist dafür paradigmatisch. ${ }^{89}$ »Ich bin [...] überzeugt, dass wir in einer durchaus endgültigen Wendung der Philosophie mitten darin stehen und dass wir sachlich berechtigt sind, den unfruchtbaren Streit der Systeme als beendigt anzusehen « (Schlick 1930/1931: 5). Er sei sich der »Tragweite« und »Inhaltsschwere« dieser Aussage bewusst, sagt Schlick, denn schließlich seien solche radikalen Erneuerungsansprüche der Philosophie überhaupt eigen: »Aber gerade die besten Köpfe unter den Denkern glaubten selten an unerschütterliche, bleibende Ergebnisse des Philosophierens früherer Zeiten und selbst klassische Vorbilder; dies erhellt daraus, dass im Grunde jedes neue System wieder ganz von vorn beginnt, dass jeder Denker seinen eigenen festen Boden sucht und sich nicht auf die Schultern seiner Vorgänger stellen mag « (ebd.: 4-5). Schlick betont, dass »fast alle großen Denker [...] eine radikale Reform der Philosophie für notwendig

89 Moritz Schlick war aufgrund eines Aufenthalts in Amerika bei der Tagung nicht persönlich anwesend. Sein Text »Die Wende der Philosophie« wurde jedoch in die Publikation mit aufgenommen. 
gehalten und selbst versucht [haben] «(ebd.: 5). Deswegen könnten im Grunde »alle Versuche, dem Chaos der Systeme ein Ende zu machen und das Schicksal der Philosophie zu wenden [...], so scheint eine Erfahrung von mehr als zwei Jahrtausenden zu lehren, nicht mehr ernst genommen werden « (ebd.). Obwohl Schlick also betont, dass die Behauptung einer grundlegenden Wende der Philosophie ein internes Merkmal der Philosophie selbst ist, gilt dies aus seiner Perspektive für die gegenwärtige Wende nicht. Diese unterscheide sich von allen vorherigen Reformversuchen und sollte durchaus ernst genommen werden. Ob Schlick dieses offensichtliche Spannungsverhältnis absichtlich herstellt, wird im Text nicht ersichtlich. Seine Behauptung jedoch enthält rhetorische Schlagkraft: Die gegenwärtige philosophische Wendung erscheint umso plausibler, gerade weil er sie von allen vergangenen Reformversuchen der Philosophie absetzt. Insofern zielt sie auf die alarmistische Behauptung von Aktualität.

Schlicks Überzeugung einer nun endgültigen - im Gegensatz zu jeder früheren - Wende der Philosophie resultiert aus der Annahme, dass die Entwicklung der Wissenschaften es inzwischen möglich gemacht hat, die Wissenschaftlichkeit der Philosophie endgültig zu beurteilen. Der wissenschaftliche Fortschritt, so die Implikation, belege, dass die herkömmliche und bestehende Philosophie wissenschaftlichen Kriterien nicht genüge. Aus der Entwicklung derWissenschaften sei ein Standpunkt emergiert, von dem aus diese Bewertung möglich geworden ist: »Daß die Lage wirklich einzigartig und die eingetretene Wendung wirklich endgültig ist, kann nur eingesehen werden, indem man sich mit den neuen Wegen bekannt macht, und von dem Standpunkte, zu dem sie führen, auf alle die Bestrebungen zurückschaut, die je als >philosophische< gegolten haben « (ebd.: $\left.5^{-6}\right)$. Ausgehend von diesem objektiven Standpunkt könne, so Schlick, der »Streit der [philosophischen] Systeme« endgültig beendet werden. Im Prinzip sei ein solcher Standpunkt bereits vorhanden. »Die Gegenwart ist, so behaupte ich, bereits im Besitz der Mittel, die einen derartigen Streit [den Streit der Systeme, MD] im Prinzip unnötig machen; es kommt nur darauf an, sie entschlossen anzuwenden « (ebd.: 5$){ }^{90}$

Woraus bestehen diese Mittel für Schlick und die Vertreter des Wiener Kreises insgesamt? »Die Wege gehen von der Logik aus (ebd.: 6). Die Logik sei - so Schlick - jene Methode, mit der die gesamte bisherige Philosophie

9o Der Aspekt der Prospektion, der im Kapitel zu Husserl herausgearbeitet wurde, wird auch in Schlicks Argumentation sichtbar: Eine grundlegende Wendung der Philosophie werde dann realisiert, so die Implikation, wenn die richtigen Mittel, d.h. die richtigen Methoden, angewendet werden; erst dann nämlich werde es möglich, alle früheren philosophischen Streitereien überblicken und beenden zu können. 
überwunden werden könne. Schlick gibt in seinem Aufsatz lediglich ein paar einordnende Hinweise dazu, was er unter »Logik« versteht. Er verweist auf Leibniz, Frege, Russell und schließlich auf Wittgenstein, der in seinem Tractatus logico-philosophicus von $1922 »$ bis zu der entscheidenden Wendung [...] vorgedrungen« sei (ebd.). ${ }^{91}$ Die Überzeugung, dass die Logik das letzte und sichere Fundament wissenschaftlicher Erkenntnis darstelle, steht somit in einem direkten Wechselverhältnis zur Überzeugung, dass eine endgültige Wende der Philosophie bevorstehe. Beide Argumente stützen sich gegenseitig: Wenn die Logik sogar die Philosophie als Ganze in noch nie da gewesener Weise erneuern kann, dann muss die Logik tatsächlich grundlegende Evidenz besitzen. Und umgekehrt: Wenn die Logik eine derart evidente Methode ist, dann ist es nicht erstaunlich, dass die Philosophie eine grundsätzliche Wende erfährt.

Für Schlick scheint also klar: Die Logik birgt das Potenzial, die gesamte Philosophie auf eine neue wissenschaftlich gültige Grundlage zu stellen. Die von Schlick in seinem Text eingeführte Trennung zwischen der Methode der Logik und der Einsicht in das Logische selbst erhärtet diese Überzeugung: »Ist nun diese Logik«, fragt Schlick, »das große Mittel, von dem ich vorhin sagte, es sei imstande, uns im Prinzip aller philosophischer Streitigkeiten zu entheben, liefert sie uns etwa allgemeine Vorschriften, mit deren Hilfe alle traditionellen Fragen der Philosophie prinzipiell aufgelöst werden können?« (ebd.). Diese Frage wird verneint. »Wäre dies der Fall, so hätte ich kaum das Recht gehabt zu sagen, daß eine völlig neue Lage geschaffen sei, denn es würde dann nur ein gradueller, gleichsam technischer Fortschritt erzielt sein, sowie etwa die Erfindung des Benzinmotors schließlich die Lösung des Flugproblems ermöglichte« (ebd.). Schlick führt die eingetretene Wende der Philosophie also nicht nur (wie Carnap) der wissenschaftshistorischen Entwicklung der Logik als Methode zu - »durch die bloße Ausbildung einer Methode kann niemals etwas so Prinzipielles geleistet werden « - , sondern »etwas ganz anderem, das durch sie wohl erst möglich gemacht und angeregt wurde, aber in einer viel tieferen Schicht sich abspielt: das ist die Einsicht in das Wesen des Logischen selber« (ebd.).

Dass die Logik ein sicheres und endgültiges Mittel für die wissenschaftliche Wahrheitsfindung ist, wird als unanfechtbare Tatsache präsentiert, die weder

91 Schlick zeigte sich wie auch andere Mitglieder des Wiener Kreises von Wittgensteins Tractatus tief beeindruckt. Er bezeichnet sich selbst als »Bewunderer« von Wittgenstein. Umgekehrt soll auch Wittgenstein Schlick geschätzt haben und ihn nicht zur »Clique«, wie er den Kreis rund um Hahn und Carnap bezeichnet hat, gerechnet haben (Sigmund 2015: 130). 
einer Legitimation noch eines weiteren Beweises bedarf. Schlick erläutert den Inhalt dieser »schlichten Einsicht« wie folgt:

Der Weg der Klarheit darüber geht von der Tatsache aus, daß jede Erkenntnis ein Ausdruck, eine Darstellung ist. Sie drückt nämlich den Tatbestand aus, der in ihr erkannt wird, und dies kann auf beliebig viele Weisen, in beliebigen Sprachen, durch beliebige willkürliche Zeichensysteme geschehen; alle diese möglichen Darstellungsarten, wenn anders sie wirklich dieselbe Erkenntnis ausdrücken, müssen eben deswegen etwas gemeinsam haben, und dies Gemeinsame ist ihre logische Form. So ist alle Erkenntnis nur vermöge ihrer Form Erkenntnis; durch sie stellt sie die erkannten Sachverhalte dar, die Form selbst aber kann ihrerseits nicht wieder dargestellt werden; auf sie allein kommt es bei der Erkenntnis an, alles übrige daran ist unwesentlich und zufälliges Material des Ausdrucks, nicht anders als etwa die Tinte, mit der wir einen Satz niederschreiben. (ebd.)

Erkennbar seien also lediglich Darstellungen und Ausdrücke. Diese Darstellungen und Ausdrücke wiederum besitzen eine logische Form. Diese Form könne selbst nicht wieder dargestellt werden, denn sie impliziere gewissermaßen die Erkenntnis selbst. Schlick geht also von einer unwiderlegbaren und überhistorischen Trennlinie zwischen wissenschaftlich Erkennbarem (bzw. Darstellbarem) und wissenschaftlich nicht Erkennbarem aus. Dass er in seiner Argumentation von einer »schlichten Einsicht« mit »Folgen allergrößte[r] Tragweite« spricht, stützt die Unwiderlegbarkeit und Evidenz seiner Aussage rhetorisch - dass nämlich die »logischen Formen« die (neuen) nicht weiter zu hinterfragenden Grundlagen der Philosophie seien. Der Grund für diese Überzeugung liegt - so wird der folgende Abschnitt deutlich machen - in einer neuen Verhältnisbestimmung zwischen Mathematik und Logik:

Bekanntlich haben die Mathematiker in den letzten Jahrzehnten neue logische Methoden entwickelt, zunächst zur Lösung ihrer eigenen Probleme, die sich mit Hilfe der überlieferten Formen der Logik nicht bewältigen ließen; dann aber hat die so entstandene Logik (siehe den Artikel von Carnap in diesem Heft) auch sonst ihre Überlegenheit über die alten Formen längst bewiesen und wird diese zweifellos bald ganz verdrängt haben. (ebd.)

Der von Schlick angesprochene Artikel von Carnap dient als Leitfaden für den nächsten Abschnitt. Er gibt Antworten auf die Frage, warum die Vertreter des Wiener Kreises von der Überlegenheit der Logik gegenüber anderen Wissenszweigen derart überzeugt waren.

\section{Die Genese der Logik aus der Mathematik}

Die neue Logik sei, schreibt Carnap in seinem Aufsatz »Die alte und die neue Logik«, »nicht mehr bloß eine philosophische Disziplin neben anderen, 
sondern wir können geradezu sagen: die Logik ist die Methode des Philosophierens" (Carnap 1930/131: 12). Carnap stellt die Logik als die (einzig noch mögliche) Methode »des Philosophierens « vor. Wie tut er das? Wie begründet er den Singularitätsanspruch der Logik?

Entscheidend in der Argumentation Carnaps ist dessen Zweiteilung der Logik in einen alten und einen neuen Bereich. Die »alte Logik « allein - gemeint ist die formale Logik aristotelisch-scholastischer Tradition - genüge nicht, die Logik als Methode der Philosophie zu etablieren. »Der Wunsch, an Stelle metaphysischer Begriffsdichtung eine streng wissenschaftliche Methode des Philosophierens zu setzen, wäre ein frommer Wunsch geblieben, wenn man als logisches Werkzeug nur das System der traditionellen Logik zur Verfügung gehabt hätte« (ebd.: 12-13). Denn die alte Logik »war gänzlich außerstande, den Ansprüchen an inhaltlichen Reichtum, formale Strenge und technische Brauchbarkeit zu genügen, die die neue Aufgabe an sie stellen mußte« (ebd.: 13). Diese alten Werke der Logik enthielten zwar »manche bemerkenswerte Überlegungen, standen aber in Bezug auf Begriffsbildung und Gründlichkeit der Analyse auf ziemlich primitiver Stufe« (ebd.). Carnap betont ausdrücklich, dass darin kein »Vorwurf gegen diese Werke« liege, sondern dass »dieser Zustand der angewandten Logik [...] durch die Unzulänglichkeit der formalen Grundlage [bedingt war] « (ebd.). Erst die neue Logik - sie sei vor ca. 50 Jahren, d.h. um 188o, entwickelt worden - enthielte die Möglichkeit, den geforderten Ansprüchen zu genügen. Diese neue Logik sei - dies ist der hier zentrale Aspekt - nicht von den Logikern alter Prägung, sondern von Mathematikern entwickelt worden:

An Stelle des unbrauchbaren alten Werkzeuges ein leistungsfähiges neues zu schaffen, hätte wohl lange Zeit erfordert. Und vielleicht darf man zweifeln, ob die Logiker aus eigenen Kräften zu diesem Werk überhaupt imstande gewesen wären. Zum Glück fand man ein Werkzeug schon vor, eine neue Logik, die in den letzten 50 Jahren entwickelt worden ist, und zwar durchweg von Mathematikern. Den Anlaß hierzu gaben Schwierigkeiten innerhalb der Mathematik; an eine philosophisch bedeutsame allgemeinere Anwendung war zunächst nicht gedacht worden. (ebd.)

Carnap führt die Entwicklung der neuen Logik also auf mathematik-interne Diskussionen ab ca. 1850 zurück. »Der wichtigste Anlaß zur Ausbildung der neuen Logik lag in der Notwendigkeit, die Grundlagen der Mathematik kritisch nachzuprüfen « (ebd.: 14). Die Mathematik hätte durch Leibniz und Newton »einen ungeheuren Aufschwung genommen« und »eine Fülle neuer Erkenntnisse gewonnen « (ebd.). »Die Sicherung der Fundamente hatte jedoch mit diesem schnellen Wachsen des Gebäudes nicht Schritt gehalten« (ebd.). 
Aus diesem Grund habe ca. um 1830 eine stärkere Bemühung um die Klärung der Grundlagen der Mathematik begonnen. »Man begnügte sich nicht damit, die verschiedenen Begriffe der Analysis auf Zahlbegriffe als die Fundamentbegriffe der Mathematik zurückzuführen, sondern stellte sich die Aufgabe einer logischen Klärung der Zahlbegriffe selbst « (ebd.: 14, Herv. MD). Aus fachinternen Problemstellungen der Mathematik, so impliziert Carnap, habe sich die Notwendigkeit einer neuen Logik entwickelt. »Diese Untersuchungen der logischen Grundlage der Arithmetik mit dem Ziel der logischen Analyse der Zahl erforderten unumgänglich ein durch Umfang und Schärfe leistungsfähiges System « (ebd.: 14). Ein besonders starker Antrieb zur Entwicklung dieses System sei durch die Untersuchungen von Peano, Frege, Whitehead, Russel und Hilbert erfolgt. Die weitere fachinterne Entwicklung, so fährt Carnap weiter, erfordere schließlich eine »gründliche Neugestaltung der Logik« selbst: »Dringender noch wurde die Notwendigkeit eines Neuaufbaus der Logik, als man gewisse Widersprüche (`Antinomien $<$ ) zunächst auf mathematischem Gebiete bemerkte, die sich aber bald als solche allgemein-logischer Natur herausstellten. Sie konnten nur durch gründliche Neugestaltung der Logik überwunden werden« (ebd.: 15).

Aus Carnaps kurzem Abriss der Entwicklung der Logik lässt sich folgendes historiografisches Modell ablesen: Um fachinterne Grundlagenprobleme der Mathematik zu lösen, haben Mathematiker ein Instrument entwickelt, welches diese Probleme zu lösen vermochte: die Logik. Mit anderen Worten, Mathematiker benötigten ein neues Instrument, um fachinterne Unklarheiten beheben zu können. Die Logik wird von Carnap also zwar als das neue Fundament der Mathematik vorgestellt, selbst aber nicht als Mathematik definiert. ${ }^{92}$ Das »große Hauptwerk der neuen Logik« sei die Principia Mathematica (19101913) von Alfred North Whitehead und Bertrand Russell. »Alle weiteren Arbeiten der neuen Logik stützen sich auf dieses Werk; sie versuchen entweder, es zu ergänzen, oder, es umzubauen « (ebd.:14). ${ }^{93}$ Die Principia Mathematica stellt für Carnap den einschneidenden Schnitt zwischen der »alten« und der »neuen« Logik dar. Mit dieser Schrift, so kann Carnap paraphrasiert werden, vollzieht sich ein Paradigmenwechsel innerhalb der Mathematik: Die Mathematik wird - mithilfe der Typenlehre - neu fundiert, d.h., sie wird nun aus logischen Axiomen hergeleitet. Dieses neue und asymmetrische Verhältnis zwischen

92 Argumentationsstrategisch wird an dieser Stelle eine Analogie zu Husserl deutlich: Die Notwendigkeit einer neuen Philosophie - hier der Logik - wird mit den fachinternen Krisen einer anderen Wissenschaft - hier der Mathematik - begründet.

93 Carnaps historische Herleitung der neuen Logik zeigt sich hier als eine Vorwegnahme der zeitlich später einsetzenden - Ursprungserzählungen der >analytischen Philosophieく. 
Logik und Mathematik wird von Carnap in verschiedenen Formulierungen wiederholt. So stellt er die Mathematik dezidiert als einen "Zweig der Logik « dar. »Es zeigt sich nämlich, daß jeder mathematische Begriff aus den Grundbegriffen der Logik abgeleitet werden kann, und daß jeder mathematische Satz [...] aus den Grundsätzen der Logik abgeleitet werden kann « (ebd.: 20). Kurz: Carnaps wissenschaftshistorische Darstellung zielt auf die Darstellung der Logik als neues Fundament der Mathematik.

In der hier eingenommenen Perspektive ist nun entscheidend, wie Carnap diese neuen Befunde aus dem Bereich der Mathematik argumentativ in den Bereich der Philosophie rücktransponiert. Die neue Logik, so behauptet er zu Beginn des Textes in programmatischer Weise, vermöge die »alte Philosophie aus den Angeln zu heben« (ebd.: 13).

In dieser neuen Logik liegt - das ist auch vielen ihrer Vertreter noch nicht bewußt - der Punkt, von dem aus die alte Philosophie aus den Angeln zu heben ist. Alle Philosophie im alten Sinne, knüpfe sie nun an Plato, Thomas, Kant, Schelling oder Hegel an, oder baue sie eine neue »Metaphysik des Seins « oder eine »geisteswissenschaftliche Philosophie« auf, erweist sich vor dem unerbittlichen Urteil der neuen Logik nicht etwa nur als inhaltlich falsch, sondern als logisch unhaltbar, daher sinnlos. (ebd.: 13)

Die neue Logik wird von Carnap also nicht nur als das neue Fundament der Mathematik dargestellt, sondern zugleich als ein Instrument, das auf andere Wissenschaften angewendet werden kann: Das neue Instrument der Logik vermag auszuweisen, dass alle bisherigen philosophischen Bemühungen »inhaltlich falsch«, »logisch unhaltbar« und daher »sinnlos« seien. Interessant ist hier, wie Carnap die in Anführungszeichen gesetzten Begriffe der »Metaphysik des Seins« bzw. der »geisteswissenschaftlichen Philosophie« verwendet: Er referiert auf Heidegger bzw. auf Dilthey, ohne deren Namen direkt zu nennen. Sowohl die Philosophie Heideggers als auch jene Diltheys erweisen sich gemäß Carnap als logisch unhaltbar, d.h. sinnlos, wenn die Methoden der neuen Logik auf sie angewendet werden.

Die Atributte »inhaltlich falsch«, »logisch unhaltbar« und »sinnlos« stehen für Carnap gleichbedeutend mit nicht wissenschaftlich. Er wendet diese Atributte auf andere Philosophien an und transponiert dadurch die neuen Befunde aus der Mathematik, nämlich, dass mit der Logik mathematische Grundlagenprobleme gelöst werden können, in den Bereich der Philosophie. Diesen Transfer begründet er aus dem Potenzial der neuen Logik selbst: »Die neue Logik unterscheidet sich von der alten [...] vor allem durch umfangreiche Gebietserweiterungen « (ebd.: 16). Als die wichtigsten »Gebietserweiterungen« nennt er die »Theorie der Beziehungssätze« und die »Theorie der variablen 
Satzfunktionen «. Es kommt an dieser Stelle nun nicht darauf an, Carnaps Argumentationen im Detail nachzuvollziehen, sondern es gilt festzuhalten, dass er in der »neuen Logik« nicht nur ein für die Mathematik fundierendes Element sieht, sondern dies zugleich auf den Bereich der Sprache und den Bereich der Wissenschaften allgemein ausweitet. Letzteres führt zu der Annahme, dass alle wissenschaftlichen Bestrebungen letztlich auf eine gemeinsame Basis zurückgeführt werden können. »Bei der Analyse der wissenschaftlichen Begriffe hat sich ergeben, daß alle Begriffe, mögen sie nun nach üblicher Einteilung zum Gebiet der Naturwissenschaften, der Psychologie oder der Sozialwissenschaften gehören, auf eine gemeinsame Basis zurückgehen $[\ldots]$ « (ebd.: 24). Potenziell, so folgt daraus, ließe sich ein abstraktes Formelsystem finden, das alle Wissenschaften logisch miteinander verbindet. »So führt die logische Analyse mit den Mitteln der neuen Logik zur Einheitswissenschaft. Es gibt nicht verschiedene Wissenschaften mit grundsätzlich verschiedenen Methoden oder gar verschiedenen Erkenntnisquellen, sondern nur die eine Wissenschaft« (ebd.: 24-25) ${ }^{94}$ Unter diese eine Wissenschaft fällt für Carnap auch die Philosophie. »Es gibt keine Philosophie als Theorie, als System eigener Sätze neben denen der Wissenschaft« (ebd.: 26). Und dennoch weist Carnap der Philosophie eine klare, sich von den Aufgaben der Einzelwissenschaften abhebende Bestimmung zu: »Philosophie betreiben bedeutet nichts anderes als: die Begriffe und Sätze der Wissenschaft durch logische Analyse klären« (ebd.). Auf der einen Seite wird die Philosophie als eine eigene Wissenschaft zurückgewiesen, um sie auf der anderen Seite als Methode für die Fundierung aller anderen Wissenschaften wieder zu rehabilitieren.

Gemäß der hier gezeichneten Argumentationslinie von Schlick und Carnap birgt die Logik einen objektiven Standpunkt, von dem ausgehend die Wissenschaftlichkeit der Philosophie ein füralle Mal beurteiltwerden kann. Hergeleitet und begründet wird dieser Befund mit der Letztgültigkeit der Logik selbst: Ausgehend von mathematikinternen Entwicklungen wird sie in den Bereich der Philosophie transponiert: Der Wiener Kreis nimmt die neuen Befunde aus der Mathematik, dass nicht mathematische, sondern logische Prinzipien die Mathematik fundierten, auf und überträgt sie auf andere Wissensgebiete.

94 Die »Einheitswissenschaft« wird vor allem von Neurath verteidigt und verfochten. Sie ist mit politischen Ansprüchen eng verbunden: »Was [...] im traditionellen Sprachgebrauch >Philosophie $<$ heißt [...], heißt später `Einheitswissenschaft<, wobei Wissenschaft als kollektive Tätigkeit begriffen wird, als bewusst geplante und betriebene, zusammenhängende, gemeinsame Arbeit der verschiedenen Spezialisten, als Beitrag zur Verbesserung des menschlichen Daseins. Wissenschaft soll nicht länger abgehoben und getrennt sein vom Alltagsleben der Masse der Bevölkerung «(Dvořák 1985: 132). 
Die Logik stelle - so die Argumentation - nicht nur die neue Grundlage der Mathematik dar, sondern zugleich die Grundlage jeder Wissenschaft, d.h. auch der Philosophie. Der Wiener Kreis begründet seinen Erneuerungsanspruch also nicht dadurch, dass er - wie Husserl oder die Kritische Theorie - der Philosophie in expliziter Weise Relevanz für die Wissenschaften und die Gesellschaft zuweist, sondern vielmehr dadurch, dass er mit der Logik einen neuen, objektiv gültigen Standpunkt behauptet. Das heißt, nicht das Herausstellen der Spezifika der Philosophie gegenüber anderen Wissenschaften, sondern die Rehabilitation der philosophischen Methode als objektiv gültiges Kriterium ist die entscheidende Strategie. Erkennbar wird diese Erneuerungsstrategie auch darin, dass die Vertreter des Wiener Kreises jeder herkömmlichen und zeitgenössischen Philosophie ihre wissenschaftliche Objektivität absprechen. Das dezidierte Bestreben, die Metaphysik als unwissenschaftlich auszuweisen, ist dafür besonders aufschlussreich.

\section{Philosophische Altlasten aus dem Weg räumen: Metaphysikkritik}

Die »wissenschaftliche Weltauffassung « wird von ihren Vertretern in einen scharfen Kontrast zur Metaphysik gestellt. Eine Formulierung aus dem »Manifest « lautet: »Die Vertreter der wissenschaftlichen Weltauffassung stehen entschlossen auf dem Boden der einfachen menschlichen Erfahrung. Sie machen sich mit Vertrauen an die Arbeit, den metaphysischen und theologischen Schutt der Jahrtausende aus dem Weg zu räumen « (Neurath/Hahn/ Carnap 1929: 314). Eines der erklärten Hauptziele des Wiener Kreises ist es laut dem »Manifest«, sowohl die Wissenschaften als auch die Philosophie von Metaphysik zu $»$ reinigen $\ll .{ }^{95}$

Carnaps Artikel »Überwindung der Metaphysik durch logische Analyse der Sprache«, in dem er seine berühmt gewordene Kritik an Heidegger entwickelt, ${ }^{96}$ ist 1931 in der »Erkenntnis« erschienen. Wie erwähnt hat bereits Wittgenstein auf die programmatische Ansage des Wiener Kreises, die Metaphysik mit Logik überwinden zu wollen, irritiert und verärgert reagiert. Diese Irritation spiegelt sich auch in der Rezeptionsgeschichte von Carnaps Schriften: Dass sein Anspruch, »die ganze Metaphysik aus der Philosophie zu verbannen,

95 Der Begriff der »Reinigung « erscheint im Manifest an zwei Stellen. »Er [Ernst Mach, MD] war besonders darum bemüht, die empirische Wissenschaft, in erster Linie die Physik, von metaphysischen Gedanken zu reinigen« (Neurath/Hahn/Carnap 1929: 302). Und: »Hier [in den Sozialwissenschaften, MD] ist zwar noch nicht derselbe Grad der Reinigung wie in der Physik erreicht; andererseits ist hier vielleicht die Reinigungsaufgabe weniger dringend « (ebd.: 313).

96 Das Schisma zwischen kontinentaler und analytischer Philosophie wird u.a. auf den >Streit` zwischen Carnap und Heidegger zurückgeführt (Friedman 200o). 
weil sich ihre Thesen nicht rational rechtfertigen lassen « (Carnap 1928: V), überzogen bis "peinlich « sei, ${ }^{97}$ wird häufig betont. Hätte man Kenntnisse über die Geschichte der Metaphysik, so etwa das Argument von Eckehart Köhler, wüsste man, dass die Natur der Metaphysik seit Aristoteles in der Akzeptanz von Prinzipien a priori liege. Die Mitglieder des Wiener Kreises aber waren keineswegs gegen solche Prinzipien, im Gegenteil: Sie fanden diese Prinzipien in logischen und empirischen `Sätzen< (Köhler 1995: 191-192). »Carnaps vermeintliche >Überwindung der Metaphysik««, so folgert Dieter Thomä, »entpuppt sich als Strategie, die auf einer zutiefst metaphysischen Voraussetzung beruht, also - horribile dictu - selbstwidersprüchlich ist. [...] Carnap bereitet nicht der >Überwindung der Metaphysik durch logische Analyse der Sprache< den Weg, sondern vielmehr der Etablierung einer eigenen Metaphysik durch ebendiese logische Analyse« (Thomä 2018: 104).

Gerade aufgrund dieser Befunde lohnt sich eine erneute Lektüre der betreffenden Stellen, jedoch gewissermaßen in umgedrehter Funktion: Der Überwindungsanspruch der Metaphysik ermöglicht es Carnap - so die These, die im Folgenden erläutert wird -, die logische Analyse als neue philosophische Position zu behaupten. Carnaps »Überwindung der Metaphysik« erweist sich in dieser Perspektive - wie Thomä es ausdrückt - allerdings selbst als eine Strategie, wiederum - wenn auch unterschwellig und nicht explizit eine eigene Metaphysik in Form ebendieser logischen Analyse zu lancieren.

Carnaps programmatisch gewordene Definition der Metaphysik - »Metaphysiker sind Musiker ohne musikalische Fähigkeit « (Carnap 1931: 240) zielt darauf, der traditionellen Philosophie eine wissenschaftlich informierte Methode abzusprechen. Die bisherige Philosophie besitze gewissermaßen kein solides Grundhandwerk. Metaphysik gebe zwar vor, Theorie zu sein, in Wirklichkeit diene sie aber lediglich zum Ausdruck des Lebensgefühls (ebd.: 238). In diesem Sinne sei sie - verkürzt gesprochen - schlechte Kunst. Metaphysiker würden für ihr Anliegen die falsche Form wählen: Sie täten besser daran, ihr Lebensgefühl mittels Literatur, Musik oder Poesie auszudrücken! Es sei zwar keineswegs falsch, so Carnap, ein Lebensgefühl ausdrücken zu wollen, nur dürfe dies nicht im Namen der Wissenschaft und der Philosophie geschehen. »Bei der Metaphysik liegt jedoch die Sache so, daß sie durch die Form ihrer Werke etwas vortäuscht, was sie nicht ist « (ebd.: 239-240). Metaphysik gebe nämlich einen theoretischen Inhalt vor, in Wirklichkeit sei dies aber eine Täuschung.

97 »Für einen Sympathisanten des Wiener Kreises, der auch nur ein wenig in der Geschichte der Metaphysik bewandert ist, ist es nachgerade peinlich[,] feststellen zu müssen, daß dieses a priori Gültige bereits bei Aristoteles, [sic!] gerade schon als das Wesen der Metaphysik angesehen wird « (Köhler 1995: 192). 
»Der Metaphysiker glaubt sich in einem Gebiet zu bewegen, in dem es um wahr und falsch geht. In Wirklichkeit hat er jedoch nichts ausgesagt, sondern nur etwas zum Ausdruck gebracht, wie ein Künstler« (ebd.: 240). Anders als der Künstler wisse der Metaphysiker nicht, »daß er sich im Gebiet der Kunst und nicht in dem der Theorie befindet « (ebd.: 240). Carnap bedient sich in seiner Argumentation eines gängigen Topos, nämlich der Trennung zwischen Kunst und Wissenschaft, um die Metaphysik als unwissenschaftlich zu disqualifizieren. Mit anderen Worten, durch seine Parallelisierung der Metaphysik mit den Künsten wird die Metaphysik aus dem Bereich der Philosophie ausgeschlossen. Metaphysische Sätze sind für Carnap Scheinsätze, d.h., sie sind konstitutiv bedeutungs- und sinnlos.

Da die Metaphysik weder analytische Sätze sagen, noch ins Gebiet der empirischen Wissenschaft geraten will, so ist sie genötigt, entweder Wörter anzuwenden, für die keine Kriterien angegeben werden und die daher bedeutungsleer sind, oder aber bedeutungsvolle Wörter so zusammenzustellen, daß sich weder ein analytischer (bzw. kontradiktorischer) noch ein empirischer Satz ergibt. In beiden Fällen ergeben sich notwendig Scheinsätze. (ebd.: 236)

Scheinsätze seien weder analytisch noch empirisch. »Die logische Analyse spricht somit das Urteil der Sinnlosigkeit über jede vorgebliche Erkenntnis, die über oder hinter die Erfahrung greifen will« (ebd.: 237). Metaphysik ist für Carnap alles Nichtempirische, das vorgibt, Erkenntnis zu sein. Erst indem er alles Nichtempirische aus dem Bereich der Philosophie auslagert, kann er seine eigene Position begründen. Er >arbeitet< folglich mit einer Strategie der Auslagerung: Indem er die Metaphysik, verstanden als traditionelle Philosophie, mit dem Argument der Unwissenschaftlichkeit aus dem Bereich des Philosophischen auslagert, argumentiert er für eine neue, nun wissenschaftliche philosophische Position.

Dass die »Überwindung der Metaphysik« als eine argumentative Strategie fungiert, Carnaps eigene Position zu begründen, wird vor allem daran deutlich, dass er den Begriff der Metaphysik als selbstevident voraussetzt. Seine Andeutungen über die Metaphysik als das Nichtempirische oder als schlechte Kunst werden nicht weiter erläutert. Erst durch seine logische Analyse wird es möglich, Sätze als metaphysisch zu entlarven. Seine These - »dass die angeblichen Sätze der Metaphysik sich durch logische Analyse als Scheinsätze enthüllen« (ebd.: 220) - setzt also bereits voraus, dass klar sei, welche Sätze zumindest potenziell - metaphysisch sind und welche nicht. ${ }^{98}$ Gerade indem

98 Hier wird eine Argumentationsstrategie deutlich, die bereits im Falle von Husserl erkennbar geworden ist und im Kapitel zur Kritischen Theorie vertieft wird: Der Wahrheitsgehalt 
Carnap nicht explizit ausführt, was unter Metaphysik zu verstehen ist oder warum Metaphysik in der Philosophie überhaupt eliminiert werden muss, nimmt sie eine wichtige Funktion in seiner Argumentation ein: Als Chiffre der Unwissenschaftlichkeit fungiert die Metaphysik als Abgrenzungsfolie für die Plausibilisierung einer neuen, nunmehr nicht metaphysischen, d.h. wissenschaftlichen Grundlegung. Die neue Logik ist dafür das entscheidende Instrument:

Der Unterschied zwischen unserer These und der der früheren Antimetaphysiker ist jetzt deutlich. Die Metaphysik gilt uns nicht als »bloßes Hirngespinst« oder »Märchen«. Die Sätze eines Märchens widerstreiten nicht der Logik, sondern nur der Erfahrung; sie sind durchaus sinnvoll, wenn auch falsch. Die Metaphysik ist kein »Aberglaube«; glauben kann man an wahre und an falsche Sätze, aber nicht an sinnlose Wortreihen. (ebd.: 232)

Liest man dieses Zitat gegen den Strich, wird abstrahierend vor allem die Unterscheidung zwischen sinnlos/sinnvoll augenscheinlich. Im Zusammenhang mit den anderen Textstellen könnte man die Textstelle also dahingehend deuten, dass ein Märchen zwar durchaus logisch sein kann, da es sich lediglich mit der Erfahrung widerspricht, die Metaphysik hingegen sei logisch falsch. Deswegen, so Carnaps Folgerung, könne sie ohne weitere Diskussion ad acta gelegt werden. Mit dem (logischen) Urteil der Sinnlosigkeit schließt Carnap bestimmte Wissenstraditionen gänzlich aus dem wissenschaftlichen und philosophischen Geltungsbereich aus. Ist etwas lediglich falsch - wie etwa das Märchen - verbleibt es doch immerhin noch im Bereich des wissenschaftlich Überprüfbaren. Ist etwas jedoch wissenschaftlich sinnlos - wie laut Carnap die Metaphysik -, ist es von vornherein aus dem Bereich der Wissenschaften und der Philosophie ausgeschlossen.

Carnap erhärtet die Grenzziehung zwischen wissenschaftlicher und metaphysischer Philosophie, wie erwähnt, mit einer Kritik an Heidegger. ${ }^{99}$

anderer zeitgenössischer Philosophien wird mit einer neu entwickelten Methode infrage gestellt. Die Strategie, die neu entwickelte Methode auf die Philosophie selbst anzuwenden und gerade dadurch eine philosophische Erneuerung zu evozieren, erweist sich als eine eminent wichtige Argumentationsstrategie (siehe dazu das Fazit, v.a. S. 192).

99 Michael Friedman versteht diese Wahl innerhalb des politischen Kontextes: »And I think there is no doubt that this social and political dimension of their disagreement represents at least part of the explanation for the circumstance that Carnap chooses precisely Heidegger for his examples of metaphysical pseudo-sentences« (Friedman 2000: 18). Thomä diskutiert im Zusammenhang mit Heideggers Urfassung von Was ist Metaphysik? dessen politisch höchst problematische Stellung im Zusammenhang mit Carnaps Kritik. »Gespiegelt auf Heidegger heißt dies [dass Carnap als Gönner versagt hat, mit seinen Sorgen aber recht hat, MD], dass er in dem Maße, wie Carnap Unrecht hat, weiterhin als 
Heideggers Philosophie sei jene »metaphysische[] Lehre, die gegenwärtig in Deutschland den stärksten Einfluss ausübt« (ebd.: 229). Carnap unterzieht einzelne Aussagen aus Heideggers Aufsatz Was ist Metaphysik? (1929) einer sprachlogischen Analyse. Diese ergibt, dass Sätze wie z.B. Das Nichts selbst nichtet inhaltsleer und sinnlos sind. Gleichzeitig betont er, dass Heidegger selbst feststelle, dass »seine Fragen und Antworten mit der Logik und der Denkweise der Wissenschaft nicht vereinbar sind « (ebd.: 232). Heidegger gehe, wie alle Metaphysiker, also durch und durch antilogisch und antiwissenschaftlich vor. Bereits deren Fragestellungen seien logisch sinnlos. Begriffe wie »Gott«, »Ursprung «, »Idee«, »das Absolute«, »das Unbedingte«, das »Unendliche«, das »Sein des Seienden«, »Ding an sich«, »absoluter Geist«, »objektiver Geist«, »Wesen «, »Ansichsein «, »Anundfürsichsein «, »Emanation «, »Manifestation «, »Ausgliederung «, »das Ich«, das »Nicht-Ich« usw. seien »spezifisch metaphysische Termini ohne Bedeutung « (ebd.: 227). Mit solchen Wörtern verhalte es sich nicht anders als mit einem willkürlich erfundenen Wort wie z.B. »babig « (dessen Bedeutungslosigkeit Carnap an einer anderen Stelle in seinem Text logisch belegt hat).

Carnaps »Überwindung der Metaphysik« geht also nicht nur mit einem direkten Angriff auf einen zeitgenössischen Philosophen - Heidegger - einher, sondern zugleich mit dem Angriff auf Kernbegriffe des abendländischen philosophischen Denkens. Seine Metaphysikkritik hat zur Folge, dass beinahe der gesamte philosophische Diskurs als metaphysisch und daher als sinnlos beurteilt wird. Carnap unterstreicht dies explizit: Sein Urteil treffe »jede spekulative Metaphysik, jede vorgebliche Erkenntnis aus reinem Denken oder aus reiner Intuition, die die Erfahrung zu entbehren glaubt« (ebd.: 237). Das Urteil treffe aber auch jede Metaphysik, die von der Erfahrung ausgehend das »außer oder hinter der Erfahrung Liegende erkennen will [...] « (ebd.), sowie jegliche Wert- oder Normphilosophie und jede Ethik oder Ästhetik. Darüber hinaus seien auch der Realismus, subjektive Idealismus, Solipsismus, Phänomenalismus und (der frühe) Positivismus vom Urteil der metaphysischen Sinnlosigkeit betroffen. Kurz: Indem Carnap gewissermaßen den gesamten philosophischen Kanon unter Metaphysik fasst, setzt er seine eigene Position von ebendiesem Kanon ab. Das Disqualifizieren von bestehenden »sinnlosen « - Philosophien und die Fundierung von neuen - »sinnvollen « Positionen sind zwei Seiten desselben Argumentationsprozesses.

Wie beschreiben die Vertreter des Wiener Kreises aber diese neue Philosophie, die in der Selbstbeschreibung der Gruppe mit der alten so gar nichts

jemand, der einen philosophischen Beitrag leistet, gelten darf, und dass er in dem Maße, wie Carnap Recht hat, aus der Philosophie herausfällt« (Thomä 2018: 101). 
zu tun hat? »Was aber bleibt dann für die Philosophie überhaupt noch übrig, wenn alle Sätze, die etwas besagen, empirischer Natur sind und zur Realwissenschaft gehören? « (ebd.). Carnaps dezidierte Antwort lautet: »Was bleibt, sind nicht Sätze, keine Theorie, kein System, sondern nur eine Methode, nämlich die der logischen Analyse (Carnap: 1928: 237).

\section{Die Philosophie als Methode bzw. Tätigkeit}

Wie also wird der Philosophie, wenn sie aus Sicht des Wiener Kreises in ihrer herkömmlichen Form doch strikt abgelehnt werden muss, dennoch ein eigenständiger und von den Fachwissenschaften unterscheidbarer Bereich zugewiesen? Wie wird die durch die Identifikation mit den Wissenschaften "gereinigte $« 100$ Philosophie neu >besetzt ? Wo an Carnaps Texten ein historiografisches Modell sichtbar wurde, das die Letztinstanz der Logik aus der Mathematik extrapoliert, wird im Folgenden an Neurath, Frank und Reichenbach die methodologische Ebene hervorgehoben: Die als neu postulierte philosophische Methode, so wird deutlich werden, habe sich an der Physik zu orientieren.

In seinem Beitrag Wege der wissenschaftlichen Weltauffassung sagt der Sozialökonom Neurath: »Die Vertreter der wissenschaftlichen Weltauffassung, die alles Erfahrbare in sich aufnimmt, verhalten sich wie Physiker « (Neurath 1930/1931: 106). Auch hier wird die Transponierung naturwissenschaftlicher Modelle in den Bereich der Philosophie offensichtlich, die bereits weiter oben deutlich geworden ist. Wo Carnap in seinem Text Die alte und die neue Logik die Gültigkeit mathematischer Erkenntnisse auf die Wissenschaften allgemein übertragen hat, geht es hier - wie Neurath selber sagt - um ein Vorbild bezüglich der Arbeitsweisen: Die Physik, so Neurath, sei eine Disziplin »mit sehr sauberen Begriffen«; Physiker seien »aktiv und gegenwartsnah, auch wenn sie sich in abstrakten Sphären bewegen. Sie bemühen sich weniger um die Geschichte ihrer Gedankengänge als um neue Einsichten, die sie in klaren Sätzen zu formulieren suchen. Bei den erlangten Ergebnissen bleibt man nicht stehen, die Formulierungen werden weiter geführt und von Jahr zu Jahr verbessert« (ebd.). Die Arbeitsweise der Physiker müsse, so impliziert Neurath, auf die Arbeitsweise der Philosophie übertragen werden. Keinesfalls gehe es aber darum, dass Philosophen physikalische Probleme behandeln sollten, im Gegenteil: Es seien die Fachwissenschaftler selbst, die üblicherweise als >philosophisch < deklarierte Probleme bearbeiten und beantworten sollen.

Dieser Anspruch auf eine Überwindung der Fächergrenzen in Bezug auf die Methode wird auch von Frank in seiner Eröffnungsansprache aufgegriffen:

100 Zum Begriff der »Reinigung« siehe Fn. 95. 
»Der Physiker ist oft zu folgender Auffassung geneigt: Wenn man tiefer gräbt, so stößt man immer auf Fragen, die der Physik nicht mehr zugänglich sind, sondern zu denen eine ganz andere Methode, die philosophische, gehört. Diese Meinung ist umso mehr verbreitet, je weniger man sich mit den Grundlagenproblemen beschäftigt « (Frank 1930/1931: 95). Diese Meinung jedoch sei falsch: »Jede tiefere Beschäftigung muß meiner Ansicht nach zu der Überzeugung führen, daß alle Probleme, die von der Physik und Mathematik aufgeworfen werden, auch nach den Methoden der exakten Wissenschaften gelöst werden können und müssen « (ebd.). Im nächsten Satz bringt Frank sein Anliegen wie folgt auf den Punkt: »Der Physiker braucht sich nicht, wenn er an einer gewissen Grenze angekommen ist, blindlings in die Arme einer veralteten Philosophie zu werfen « (ebd.). Frank kritisiert somit Physiker dahingehend, dass diese bestimmte Fragen fälschlicherweise aus ihrem Fachgebiet auslagerten. Die Physik könne zwar an ihre Grenzen stoßen, es sei aber nicht wie eine verbreitete Meinung sage - die Aufgabe von Philosophen, die Fragen, die jenseits dieser Grenze liegen, zu beantworten, sondern es sei die Aufgabe der Fachwissenschaftler selbst, diese Grenze zu überwinden. Es gebe »heute schon genug Bestrebungen, auch die Grundbegriffe und -probleme nach rein wissenschaftlichen Methoden zu bearbeiten « (ebd.). Frank verknüpft diese Auffassung mit einem Ziel der gesamten »Tagung für Erkenntnislehre der exakten Wissenschaften«:

Und wenn diese Tagung so ausfällt, wie sie, meiner Ansicht nach, einen Sinn für Mathematiker und Physiker haben kann, so muß sie in ihnen nicht die Überzeugung erwecken, daß man eine ihnen fremde Philosophie braucht, aber anstatt dessen einen Zweig der exakten Wissenschaften, der die Grundprobleme mit derselben Strenge, mit derselben erbarmungslosen Forderung nach Klarheit behandelt, die der Physiker und Mathematiker im Inneren seiner Wissenschaft von jeher zu fordern gewohnt ist. (ebd.)

Die Tagung soll laut Frank zeigen, dass nicht eine der Mathematik und Physik äußerliche Philosophie für die vorerst unerklärbaren Probleme der Physik zuständig ist, sondern es seien Mathematiker und Physiker selbst, die diese Aufgaben zu lösen haben. Wie weiter oben bereits thematisiert, wird ein ähnlicher Gedanke auch von Reichenbach in seiner Ansprache »Zur Einführung« aufgeworfen: Für die Philosophie seien einzig jene Erkenntnisse relevant, die aus den Fachwissenschaften resultierten. Diese aus den Einzelwissenschaften gewonnenen Erkenntnisse sollen laut Reichenbach in einer neuen Zeitschrift, der »Erkenntnis«, ein Organ finden (Reichenbach 1930/31: 1). Sowohl in Neuraths als auch in Franks und Reichenbachs Bemerkungen wird also die Ansicht deutlich, dass es zwar durchaus genuin philosophische Erkenntnisse 
gibt, dass diese aber ausgehend von den fachwissenschaftlichen Methoden selbst bearbeitet und behandelt werden sollen. Die Beispiele verdeutlichen, dass die Fächergrenzen zwischen der Philosophie und den Fachwissenschaften aufgelöst werden, ohne die Relevanz genuin >philosophischer Erkenntnisse< infrage zu stellen.

Worin genau diese aus den Fachwissenschaften stammenden philosophischen Erkenntnisse jedoch bestehen bzw. wie sie sich von anderen Erkenntnissen unterscheiden, wird nicht weiter thematisiert. Argumentativ wird also lediglich der >Ort<, wo diese aufzufinden sind, verlagert: Im Verständnis der herkömmlichen Philosophie seien sie in der Fachwissenschaft Philosophie aufzufinden, im Verständnis der neuen - wissenschaftlichen Philosophie hingegen in den (naturwissenschaftlichen) Fachwissenschaften. Negiert wird also lediglich die Möglichkeit der Philosophie als einheitliche Wissenschaft (»Systemphilosophie«) und nicht etwa die Existenz genuin philosophischer Erkenntnisse.

Der zentrale Unterschied zwischen der herkömmlichen Philosophie und der neuen wissenschaftlichen Weltauffassung sei - so schreiben auch die Autoren des »Manifests « -, dass die wissenschaftliche Weltauffassung ${ }^{101}$ keinen eigenen Gegenstand und kein eigenes Erkenntnisobjekt besitze, das sie untersuchen könnte: »Rückblickend wird uns nun das Wesen der neuen wissenschaftlichen Weltauffassung im Gegensatz zur herkömmlichen Philosophie deutlich: Es werden nicht eigene >philosophische Sätzeく aufgestellt, sondern nur Sätze geklärt; und zwar Sätze der empirischen Wissenschaft, wie wir es bei den verschiedenen, vorhin erörterten Problemgebieten [Grundlagen der Arithmetik, Physik, Geometrie, Biologie, Psychologie und Sozialwissenschaften, MD] gesehen haben« (Neurath/Hahn/Carnap 1929: 314). Die >Auflösung der Philosophie in die Fachwissenschaften und die Konzeptualisierung der Philosophie als einer Methode im Dienste dieser Wissenschaften sind zwei Seiten derselben Medaille.

Dass die Vertreter des Wiener Kreises philosophische Erkenntnisse mit den Ergebnissen aus den Fachwissenschaften identifizieren, ohne dass aus ihrer Sicht damit einherginge, dass die Philosophie ihre Eigenständigkeit einbüßte,

101 Dass die Vertreter des Wiener Kreises von »Weltauffassung « und nicht etwa von »Weltanschauung« sprechen, ist zentral. Im Zusammenhang mit seiner Metaphysikkritik schreibt Carnap: »Hierbei [wie sich in Stil und Art des Kunstwerkes das Lebensgefühl kundgibt, MD] wird häufig der Ausdruck >Weltanschauung gebraucht; wir vermeiden ihn lieber wegen seiner Zweideutigkeit, durch die der Unterschied zwischen Lebensgefühl und Theorie verwischt wird, der für unsere Analyse gerade entscheidend ist« (Carnap 1931: 239). 
wird - paradoxerweise - durch folgende Aussage Schlicks offenkundig: »Hier dürfen wir noch hinzufügen, dass die ganz entscheidenden, epochemachenden Fortschritte der Wissenschaft immer von dieser Art sind, daß sie eine Klärung des Sinnes der fundamentalen Sätze bedeuten und daher nur solchen gelingen, die zur philosophischen Tätigkeit begabt sind; das heißt: der große Forscher ist immer auch Philosoph «(Schlick 1930/31: 10).

Die Argumentationsstrategien der Vertreter des Wiener Kreises zielen auf eine neue Absteckung der Geltungsbestimmungen der Philosophie: In den Geltungsbereich der Philosophie eingeschlossen werden lediglich wissenschaftlich bereits erhärtete Erkenntnisse. Ob es sich dabei um Erkenntnisse aus den Natur- oder den Geisteswissenschaften handelt, spielt, wie etwa Reichenbach sagt, nur eine untergeordnete Rolle.

Solange die Naturwissenschaften wie bisher den weitaus größten Teil an Erkenntnissen in die Philosophie hereintragen, solange werden sie deshalb den Schwerpunkt der Zeitschrift bestimmen; aber an sich scheint uns eine Befruchtung der Philosophie durch die Geisteswissenschaften, die wir überhaupt nur in arbeitstechnischem Sinne von den Naturwissenschaften abtrennen möchten, in gleicher Weise möglich, und wir hoffen, von solcher Philosophie der Geisteswissenschaften ebenfalls Zeugnisse bringen zu können. (Reichenbach 1930/ 31: 1-2)

Die Trennlinie, die Reichenbach hier für die Geltungsbestimmung der Philosophie produktiv macht, liegt also nicht zwischen verschiedenen Disziplinen, sondern zwischen wissenschaftlichen und nicht wissenschaftlichen Befunden. Philosophisch relevant, so die Implikation, sind alle wissenschaftlich erhärteten Erkenntnisse.

An dieser Stelle erhalten die Erneuerungsstrategien des Wiener Kreises ihre Form: Der Philosophie bzw. ihrer Methode (der logischen Analyse) wird die Aufgabe zugewiesen, die Grenzen zwischen wissenschaftlich und nicht wissenschaftlich endgültig ziehen zu können. Durch ihre Identifizierung der philosophischen Tätigkeit mit einer mathematisch-physikalischen Methode grenzen die Vertreter des Wiener Kreises die Philosophie nicht nur von allen anderen Wissenschaften ab, sondern installieren sie darüber hinaus als Fundament dieser Wissenschaften. Gerade indem die Vertreter des Wiener Kreises vor allem durch ihre Metaphysikkritik - die philosophische Tätigkeit auf den Bereich der Wissenschaften einschränken, rehabilitieren sie argumentativ ein traditionelles Merkmal der Philosophie, nämlich über alles, d.h. über alles Wissenschaftliche, sprechen zu können. Durch die strikte Ablehnung der Philosophie als eigenes Fach und ihre Einschränkung auf eine Methode oder 
Tätigkeit wird ihr Geltungsbereich gewissermaßen auf alle Wissenschaften übertragen.

Dieser Erneuerungsanspruch, der mit der Identifikation der philosophischen Tätigkeit mit der Logik legitimiert wird, wird von Schlick wie folgt auf den Begriff gebracht: »Erkennbar ist alles, was sich ausdrücken lässt, und das ist alles, wonach man sinnvoll fragen kann. Es gibt daher keine prinzipiell unbeantwortbaren Fragen, keine prinzipiell unlösbaren Probleme «102 (Schlick 1930/31: 7). Das »Wesen der Dinge« durch Erkenntnisse ausdrücken zu wollen, so Schlick, sei schlichter Unsinn. »Es war einer der schwersten Irrtümer vergangener Zeiten, dass man glaubte, den eigentlichen Sinn und letzten Inhalt wiederum durch Aussagen zu formulieren, also in Erkenntnissen darstellen zu können: es war der Irrtum der >Metaphysik« (ebd.: 8-9). Das Streben der Metaphysiker sei seit jeher auf ein »widersinniges Ziel« gerichtet, nämlich darauf, »das Unsagbare zu sagen«103 (ebd.: 9).

Die Grenzziehung zwischen dem >Sagbaren < und dem >Unsagbaren < und die Rückführung des `Sagbaren` auf die Wissenschaft und die Sprache wird durch die Transponierung von Erkenntnissen und Modellen aus den exakten Wissenschaften in den Bereich der Geisteswissenschaften hergeleitet und begründet. Der Anwendungs- und Geltungsbereich der Philosophie wird neu abgesteckt. Erst innerhalb dieser wissenschaftlich aktualisierten Anwendungsbereiche

102 Schlick soll Wittgenstein, wie erwähnt, regelrecht verehrt haben. Es sei vor allem ihm zuzuschreiben, dass Wittgensteins Tractatus in den Sitzungen des Wiener Kreises über zwei Semester Satz für Satz diskutiert worden sei. Folgende Anekdote wird kolportiert: »Aber nicht alle verfielen Wittgensteins Zauber: So blieb etwa Otto Neurath dagegen völlig immun. Er witterte Metaphysik hinter den meisten Sätzen des Traktats, und rief das immer wieder lautstark in die philosophische Runde. Schließlich ersuchte ihn Schlick, doch der Kürze halber nur mehr >M! zu rufen. Neurath konterte, dass es noch kürzer wäre, >non-M! zu rufen, und zwar nach jenen Sätzen Wittgensteins, die nicht metaphysische waren« (Sigmund 2015: 119).

103 Diese Passage erinnert an einen der wohl berühmtesten Sätze der Philosophie des 20. Jahrhunderts, nämlich an Wittgensteins Schlusssatz des Tractatus: »Was sich überhaupt sagen lässt, lässt sich klar sagen; und wovon man nicht sprechen kann, darüber muss man schweigen «(Wittgenstein 1984 [1922]: 85). Die Unterschiedlichkeit in den Fokussierungen von Wittgensteins und Schlicks Aussagen kann als Widerspiegelung der ambivalenten Rolle gelesen werden, die Wittgenstein im Wiener Kreis einnimmt: Wo Wittgensteins Satz die Trennung zwischen »Sagbarem « und »Unsagbarem« fokussiert, die Relevanz des »Unsagbaren« aber keineswegs infrage stellt, fokussiert Schlick auf die Begründung des »Sagbaren« als Erkenntnis und auf die Gleichsetzung des »Unsagbaren« mit Metaphysik. Was bei Wittgenstein gewissermaßen wertneutral daherkommt, ist bei Schlick mit einem Imperativ verbunden: Metaphysik muss überwunden, wissenschaftliche Erkenntnis aber gefördert werden. 
wird der Philosophie ihre tradierte Rolle als »Alpha und Omega aller wissenschaftlicher Erkenntnis« (Schlick 1930/31: 8) zugestanden und zugesprochen.

\section{Kein eigenes Fach, aber Königin aller Fächer!}

Die Ablehnung der Philosophie als eigenständige Wissenschaft geht im Wiener Kreis so weit, dass die Nichtverwendung des Begriffes Philosophie zur Debatte stand. »Manche Vertreter der wissenschaftlichen Weltauffassung wollen, um den Gegensatz zur Systemtheorie noch stärker zu betonen, für ihre Arbeit das Wort >Philosophie< überhaupt nicht mehr anwenden « (Neurath/Hahn/ Carnap 1929: 314). Die Auffassung, dass es sich bei der Philosophie um eine Art Universal- oder Grundwissenschaft handele, wird verworfen: »Es gibt keine Philosophie als Grund- oder Universalwissenschaft neben oder über den verschiedenen Gebieten der einen Erfahrungswissenschaft; es gibt keinen Weg zu inhaltlicher Erkenntnis neben dem der Erfahrung; es gibt kein Reich der Ideen, das über oder jenseits der Erfahrung stände « (ebd.). Eine Sonderstellung der Philosophie gegenüber den anderen Wissenschaften wird abgelehnt: Die Philosophie sei den Wissenschaften gegenüber weder über- noch untergeordnet, sondern gehe - im Gegenteil - ganz in den Einzelwissenschaften auf. Diese Ablehnung ist umso bemerkenswerter, weil die Vertreter des Wiener Kreises in der Logik ja genau dasjenige Potenzial erkennen, das sie der herkömmlichen Philosophie, d.h. der Metaphysik, absprechen, nämlich das Potenzial, Erkenntnisse letztgültig klären zu können. Die Zurückweisung der Metaphysik ist in diesem Sinne als ein Frontalangriff auf die Philosophie und allgemein als eine Provokation zu verstehen.

Die Ablehnung der herkömmlichen und die Installierung einer neuen Philosophie sind zwei Seiten derselben Argumentationsstrategie. Die Vertreter des Wiener Kreises verteidigen dadurch die Möglichkeit letztgültiger, strikt wissenschaftlicher Erkenntniskriterien, ohne dass dabei der Bereich der Metaphysik tangiert wird. Schlick schreibt in seinem Beitrag: »Wir erkennen jetzt in ihr [der Philosophie, MD] - und damit ist die große Wendung in der Gegenwart positiv gekennzeichnet - anstatt eines Systems von Erkenntnissen ein System von Akten; sie ist nämlich diejenige Tätigkeit, durch welche der Sinn der Aussagen festgestellt oder aufgedeckt wird « (Schlick 1930/31: 8). Die »neue«, nun wissenschaftliche Philosophie, so Schlick, habe die Aufgabe, die Sinnhaftigkeit der Wissenschaft zu garantieren. »Durch die Philosophie werden Sätze geklärt, durch die Wissenschaften verifiziert. Bei diesen handelt es sich um die Wahrheit von Aussagen, bei jener aber darum, was die Aussagen eigentlich meinen« (ebd.). Weil »Inhalt, Seele und Geist der Wissenschaften« natürlich in dem stecke, was mit ihren Sätzen letztlich gemeint ist, sei die philosophische Tätigkeit der Sinngebung aller Anfang und aller Ende wissenschaftlicher Tätigkeit. 
Dass die philosophische Tätigkeit das Alpha und Omega aller wissenschaftlichen Erkenntnis ist, habe man geahnt, »wenn man sagte, die Philosophie liefere sowohl die Grundlage wie den Abschluss des Gebäudes der Wissenschaften; irrig war nur die Meinung, daß das Fundament von >philosophischen Sätzen gebildet werde (den Sätzen der Erkenntnistheorie), und daß der Bau auch von einer Kuppel philosophischer Sätze (genannt Metaphysik) gekrönt werde« (ebd.). Laut Schlick ist die Philosophie also zwar Anfang und Ende aller wissenschaftlichen Erkenntnis, selbst jedoch sei sie keine Wissenschaft. ${ }^{104} »$ Jede Wissenschaft [...] ist ein System von Erkenntnissen, d.h. von wahren Erfahrungssätzen; und die Gesamtheit der Wissenschaften, mit Einschluss der Aussagen des täglichen Lebens, ist das System der Erkenntnisse; es gibt nicht außerhalb seiner noch ein Gebiet > philosophischer Wahrheiten, die Philosophie ist nicht ein System von Sätzen, sie ist keine Wissenschaft « (ebd.: 7-8). Die traditionelle Philosophie jedoch, so impliziert Schlick, behaupte genau dies; sie gebe vor, eine Wissenschaft, d.h. ein System von sinnvollen Sätzen, zu sein, in Wirklichkeit sei sie das aber nicht. »Was ist sie aber dann?«, fragt Schlick in Bezug auf die »neue« Philosophie und antwortet: »Nun, zwar keine Wissenschaft, aber doch etwas so Bedeutsames und Großes, daß sie auch fürder, wie einst, als Königin der Wissenschaften verehrt werden darf; denn es steht ja nirgends geschrieben, daß die Königin der Wissenschaften selbst auch eine Wissenschaft sein müsste (ebd.: 8). In der hier eingenommenen Lesart trägt diese Wendung Schlicks nahezu absurde Züge. Allerdings zeigt sie anschaulich, wie der Wiener Kreis - gerade auch durch seine Ablehnung der traditionellen Metaphysik - die Philosophie als eine metaphysische Wissenschaft rehabilitiert. In Michael Festls Worten: »Die Philosophie bestimmt, was Wissenschaft überhaupt sein kann und was die Wahrheitskriterien einer bestimmten Wissenschaft sind (Festl 2018: 272-273). Das bedeutet umgekehrt auch, dass die Philosophie als unabhängige Instanz vorgestellt wird. Alfred Jules Ayer, dessen Buch Language, Truth and Logic (1936) wesentlich zur Popularisierung des Logischen Empirismus (vor allem in den USA) beigetragen hat, beschreibt diesen Aspekt wie folgt:

[...] the propositions of philosophy are not factual, but linguistic in character that is, they do not describe the behavior of physical, or even mental, objects; they express definitions, or the formal consequences of definitions. Accordingly, we may say that philosophy is a department of logic. For we shall see that the characteristic mark of a purely logical enquiry is that it is concerned with the

104 Argumentationsstrategisch ist hier der Unterschied zu Husserl aufschlussreich: Husserl positioniert die Philosophie explizit als eigenständige Wissenschaft, wohingegen die Vertreter des Wiener Kreises der Philosophie diesen Status absprechen. 
formal consequences of our definitions and not with questions of empirical fact. It follows that philosophy does not in any way compete with science. The difference in type between philosophical and scientific propositions is such that they cannot conceivably contradict one another. And this makes it clear that the possibility of philosophical analysis is independent of any empirical assumptions. That it is independent of any metaphysical assumptions should be even more obvious still. (Ayer 1967 [1936]: 57, Herv. MD)

Es mutet fast ironisch an, dass der Wiener Kreis, der vordergründig den unabhängigen Status der Philosophie attackiert, der Philosophie schließlich diesen Status wieder zuspricht. Die argumentative Eliminierung der Fachwissenschaft Philosophie und die Rehabilitation der Philosophie als Königin unter den Wissenschaften und für die Wissenschaften sind zwei Seiten desselben Argumentationsprozesses.

\section{Allgemeingültigkeit}

Die Rückführung des philosophischen Anwendungs- und Geltungsbereichs auf alles >Sagbare<, d.h. auf sprachliche und wissenschaftstheoretische Aspekte, ermöglicht - so ist deutlich geworden - gleichzeitig eine Aktualisierung der Philosophie als grundlegende Instanz. Die Logik wird als unabhängig sowohl von den Fachwissenschaften als auch von der Metaphysik vorgestellt, gerade weil sie aus den exakten Wissenschaften extrapoliert wird, denen wiederum ein absoluter Wahrheitsanspruch zukommt.

Die neue Funktion der Philosophie liegt gemäß diesem Erneuerungsanspruch darin, alle anderen Wissenschaften logisch miteinander zu verbinden. Diese Idee einer »Einheitswissenschaft «105 wiederum setzt den Anspruch auf Allgemeingültigkeit und in diesem Sinne Objektivität voraus. Reichenbach etwa führt in seiner Ansprache »Zur Einführung« den »Verzicht auf Allgemeingültigkeit« auf eine »Einengung des Blicks« zurück, der »die Unterschiede philosophischer Systeme in perspektivischer Verzerrung zu groß sieht und die Menge des bestehenden gemeinsamen Erkenntnisgutes zu gering veranschlägt« (Reichenbach 1930/31: 2). Würde man sich auf dasjenige konzentrieren, was man bereits weiß, »so bemerkt man auch auf

105 Die »Einheitswissenschaft« war ein umstrittenes Konzept, das, wie erwähnt, vor allem von Neurath verfochten wurde. Kurt Lewin, ein Vertreter der Berliner Gruppe, spricht sich explizit gegen die »Einheitswissenschaft « aus: »Ich habe nicht die Absicht, aus der Geschichte der Physik deduktiv zu schließen, was die Biologie tun >sollk. Denn ich bin nicht der Meinung, dass es letzten Endes nur eine einzige empirische Wissenschaft, die Physik, gibt, auf die alle übrigen zurückgehen« (Lewin 1930/31: 423). Siehe dazu auch Peters 2016: 53-54. 
philosophischem Gebiet eine weitreichende Übereinstimmung « (ebd.). Wenn man sich aber wie die Systemphilosophie auf dasjenige konzentriere, was man noch nicht weiß, habe man »nur zu reichlich Gelegenheit, Richtungen und Standpunkte in die Welt zu setzen « (ebd.). Je weiter man sich von der Philosophie in die Einzelwissenschaften hineinbewege, so Reichenbach, »und den Gedankengehalt aufzudecken versucht, der von den Forschern im Zusammenhang ihrer fachwissenschaftlichen Resultate als philosophischer Rahmen mitgedacht wird«, umso mehr werde man gemeinsam erarbeitete Wissensbestände finden. Dann werde man »nicht länger zweifeln können, daß es auch in der Philosophie eine objektive Erkenntnis gibt« (ebd.). Der Verzicht auf Allgemeingültigkeit ist gemäß Reichenbach lediglich einer Gewöhnung geschuldet: »Man hat sich in der Philosophie so sehr an Spaltung in Richtungen und Systeme gewöhnt, daß man darüber den Gedanken an eine einheitliche philosophische Wissenschaft nahezu aufgegeben hat und es als eine Besonderheit der Philosophie hinzustellen versuchte, daß es in ihr nur Lehrmeinungen, aber keine Lehre, daß es nur Standpunkte, aber keine Erkenntnis gibt« (ebd.). Reichenbach als Vertreter der Berliner Gruppe unterscheidet sich (wie weiter oben gezeigt) von den Vertretern des Wiener Kreises zwar hinsichtlich seines Philosophieverständnisses, nicht aber hinsichtlich seines Anspruchs auf Objektivität. Sowohl die Berliner Gruppe als auch der Wiener Kreis argumentieren in Übereinstimmung für die Möglichkeit nicht nur wissenschaftlicher, sondern davon ausgehend auch philosophischer Allgemeingültigkeit, Objektivität und Universalität.

Aus Sicht des Wiener Kreises und der Berliner Gruppe widerspricht dieser Anspruch auf Allgemeingültigkeit der Einsicht in die grundsätzliche Begrenztheit von Erkenntnis nicht. »Unser Denken ist ein Werkzeug, es ist abhängig von sozialen und geschichtlichen Verhältnissen. Man sollte das nie vergessen. Wir können nicht gleichzeitig den Ankläger und den Angeklagten spielen und uns überdies noch auf den Stuhl des Gerichts setzen« (Neurath 1930/31: 123). Für die wissenschaftliche Weltauffassung sei es entscheidend, »die Enge und Begrenztheit der Erkenntnis zum Bewußtsein zu bringen [...], weil sonst die Gefahr besteht, daß man durch das Postulat der völligen Bestimmtheit ein neues Idol schafft, das dann an die Stelle des alten Apriori, des Unendlichen und der Gottheit treten würde « (ebd.). Carnap will die neue Logik explizit nicht als Apriori verstanden wissen, dem ein quasi göttlicher Status zukommt. »Wo früher der Priester oder Philosoph stand, würde der Professor stehen. Von solchen übereilten Postulaten müssen wir uns fernhalten « (ebd.). Auch Reichenbach betont diesen Punkt in seinem später verfassten Buch Der Aufstieg der wissenschaftlichen Philosophie: 
Im Gegensatz dazu [zu spekulativen Systemen, MD] weigert sich die wissenschaftliche Philosophie, irgendeine Erkenntnis über die physikalische Welt als absolut sicher anzusehen. Die Gesetze der Logik und der Mathematik sind das einzige Gebiet, auf welchem Gewissheit erreicht werden kann [...]. Die wissenschaftliche Philosophie hat den Plan, ethische Vorschriften zu geben, völlig aufgegeben und betrachtet moralische Ziele als Ergebnisse von Willensakten, nicht aber als Gegenstand der Erkenntnis. (Reichenbach 1977 [1951]: 346)

Der Erneuerungsanspruch des Wiener Kreises erhält seine Wirkkraft dadurch es wird hier erneut deutlich -, dass er den Absolutheitsanspruch der herkömmlichen Philosophie ablehnt, den exakten Wissenschaften aber zugleich die Fähigkeit zuspricht, absolute Gewissheit im Prinzip erreichen zu können. Aber durch die Identifikation nun auch der Philosophie mit dieser Fähigkeit wird ebendiese gleichfalls als Möglichkeit rehabilitiert, absolutes und objektives Wissen erreichen zu können. Die Vertreter des Wiener Kreises wehren sich gegen die Auffassung, dass die Philosophie einen eigenen Zugang zur Wahrheit besitze, und forcieren die Möglichkeit, dass mit einer auf logischen Prinzipien aufbauenden Philosophie die Wahrheit gefunden werden kann.

\subsection{Zwischenfazit: Fundierung durch Identifikation}

Im Erneuerungsanspruch des Wiener Kreises wird eine Kombination verschiedener Strategien manifest: Mit der Abgrenzung gegenüber der Metaphysik auf der einen Seite und der Identifikation mit den philosophischen Methoden der exakten Wissenschaften auf der anderen Seite begründen die Vertreter des Wiener Kreises argumentativ ein neues Fundament der Philosophie. Die in vielen traditionellen philosophischen Konzepten enthaltene Vorstellung der Philosophie als Hüterin von Objektivität und Universalität wird über eine Identifizierung der philosophischen Position mit den exakten Wissenschaften legitimiert. Der gewissermaßen aus einem singulären Geist (der Metaphysik) stammende Objektivitätsanspruch wird zunächst zwar abgelehnt, um ihn dann jedoch durch eine Identifikation der Philosophie mit Modellen aus der Mathematik und Physik zu rehabilitieren. Eine Pointe der Neupositionierung des Wiener Kreises liegt darin, dass hier metaphysische Standpunkte mit den Mitteln der >harten`Wissenschaften wie Mathematik und Physik begründet werden. Allgemeingültig und objektiv sind aus Sicht der Vertreter des Wiener Kreises nicht philosophische Systeme, sondern das Gesamtsystem der Wissenschaften, dessen Fundierung Aufgabe der Philosophie ist. Die Philosophie wird folglich als unverzichtbar für das Gesamtsystem der Wissenschaften 
vorgestellt, und ihr Selbstbild als Königin der Wissenschaften wird gewissermaßen durch die Hintertüre der Wissenschaftstheorie reaktiviert.

Werden die Neupositionierungsstrategien des Wiener Kreises mit jenen von Husserl verglichen, zeigen sich sowohl Analogien als auch Unterschiede. Wie Husserl proklamieren auch die Vertreter des Wiener Kreises eine grundsätzliche Wende der Philosophie. Wie Husserl begründen sie diese Wende mit den Entwicklungen der Wissenschaften. Wo Husserl jedoch die Wissenschaftlichkeit der Philosophie quasi als immanente Notwendigkeit voraussetzt, weil ansonsten die Philosophie ihre Glaubwürdigkeit und dadurch ihre Wirkmacht verlieren würde, wird die Wissenschaftlichkeit der Philosophie von den Vertretern des Wiener Kreises demgegenüber mit dem Fortschritt der Wissenschaften begründet. Während es für Husserl gilt, den neuen philosophischen Standpunkt im Zusammenhang mit den gesamtwissenschaftlichen Entwicklungen kontinuierlich (prospektiv) zu entwickeln, ist er aus Sicht der Vertreter des Wiener Kreises bereits da: Er liegt in der Logik. Kurz: Für Husserl ist eine Umwendung der Verhältnisse in der Philosophie als anstehende historische Neuorientierung möglich, für den Wiener Kreis ist diese bereits vollzogen.

Sowohl Husserl als auch der Wiener Kreis wollen die Philosophie neu fundieren.Jedoch dominiert im Fundierungsanspruch des Wiener Kreises nicht wie bei Husserl eine Strategie des >Lücken- und Fehlerausweisens<, sondern eine der >Auslagerung : Durch die Auslagerung der Metaphysik (und dadurch beinahe der gesamten philosophischen Tradition) aus dem Bereich des Wissenschaftlichen wird der neue - mit der neuen Logik identifizierte - Standpunkt begründet. In beiden Strategien wird die Philosophie also gewissermaßen »gereinigt «; 106 bei Husserl von den Fehlern der empirischen Psychologie, beim Wiener Kreis von der gesamten Metaphysik und den damit verbundenen Traditionen.

In beiden Erneuerungsansprüchen ist das Selbstbild der Philosophie als Fundament aller Wissenschaften enthalten. Sowohl bei Husserl als auch beim Wiener Kreis wird also die Deutungsmacht der Philosophie im Zusammenhang mit den anderen Wissenschaften hergeleitet, wobei eine Hierarchisierung sichtbar wird: Während Husserl den Machtanspruch der Philosophie damit begründet, dass der Philosophie per se ein privilegierterer Zugang zur Wahrheit inhärent sei - anders als in anderen Wissenschaften -, argumentieren die Vertreter des Wiener Kreises mit dem Wahrheitsanspruch der Logik selbst: Da die Logik mit einem wissenschaftlichen Wahrheitsanspruch überhaupt

106 Zum Begriff der »Reinigung« siehe Fn. 95. 
gleichgesetzt wird, kommt ihr gleichzeitig die Macht über alle anderen Wissenschaften zu.

Beide Erneuerungsansprüche reagieren auf philosophieinterne Krisen. Husserls Erneuerungsanspruch kann als eine Reaktion auf die Bedrohung verstanden werden, die Philosophie könne aufgrund der potenziellen empirischen Vermessbarkeit des menschlichen Geistes überflüssig werden. Der Erneuerungsanspruch des Wiener Kreises wiederum kann als eine Reaktion auf die Bedrohung verstanden werden, dass die Philosophie ihren objektiven Status aufgrund des wissenschaftlichen Fortschritts verlieren könnte. In beiden Beispielen zeigte sich, dass andere Wissenszugänge für die Begründung der je neuen Position konstitutiv sind: Husserl kann sein Veto, dass rein empirische Argumente für die Erfassung des Bewusstseins ungenügend seien, erst durch die Aufnahme psychologischer Aspekte in seine Argumentation ausweisen. Am Beispiel des Wiener Kreises wiederum ist deutlich geworden, dass Carnap die Evidenz der Logik ausgehend von Diskussionen herleitet und begründet, die aus der Mathematik stammen.

In beiden Beispielen sind sowohl Abgrenzungs- als auch Identifikationsstrategien aufzufinden: Während Husserl sein eigenes Philosophieverständnis mit der philosophischen Tradition identifiziert, grenzen die Vertreter des Wiener Kreises ihr Philosophieverständnis von ebendieser Tradition ab. In beiden Erneuerungsansprüchen ist die Überzeugung vorhanden, dass sich ausgehend vom je neuen Standpunkt ein unendlich weites Arbeitsfeld eröffnet: Für Husserl liegt es in der Phänomenologie, für die Vertreter des Wiener Kreises in der Analyse der Sprache. In beiden Erneuerungsansprüchen wird eine Konzeptualisierung der Philosophie als einer neuen Methode sichtbar: Während jedoch Husserl seine Phänomenologie als »Wesensanalyse vom Bewußtsein « als eine Wissenschaft - und zwar als erste Wissenschaft - verstanden wissen möchte, lehnt der Wiener Kreis diesen Anspruch ab, um die Logik als das Fundament aller Wissenschaften zu behaupten. An die Stelle der Fachwissenschaft Philosophie tritt hier das Bestreben, alle anderen Wissenschaften auf ihre logische Struktur und ihren gemeinsamen Zusammenhang hin zu bestimmen, d.h., die Funktion der Philosophie wird explizit als eine Methode für die anderen Fachwissenschaften konzipiert.

Sowohl Husserl als auch die Vertreter des Wiener Kreises stecken das Anwendungsgebiet für die Philosophie argumentativ neu ab: Während Husserl das Bewusstsein bzw. das Sein (Phänomene) durch eine Abgrenzung gegenüber der empirischen Psychologie als privilegierten philosophischen Anwendungsbereich konstituiert, konstituieren die Vertreter des Wiener Kreises die Struktur der Wissenschaften und der Sprache als neues philosophisches Arbeitsgebiet. Durch das Argument, dass lediglich wissenschaftlich bereits 
erhärtete Erkenntnisse für die Philosophie relevant sein können, beschränkt der Wiener Kreis den Anwendungsbereich der Philosophie auf den Bereich der Wissenschaften und der Sprache. Die Rolle, die die Philosophie innerhalb dieses Anwendungsbereichs aus Sicht des Wiener Kreises einnehmen soll, nämlich die Klärung, ob Erkenntnisse wahr oder falsch, sinnvoll oder sinnlos sind, bedeutet die Reaktivierung einer traditionellen Aufgabe der Philosophie: endgültige Erkenntniskriterien zu bestimmen. In beiden Traditionen wird also das Gebiet des Philosophischen neu abgesteckt und neu definiert. Gleichzeitig sind beide Definitionen von einer Offenheit, im Prinzip von einer Unbegrenztheit bestimmt: Sowohl Husserls Phänomenologie als auch die analytische Sprachphilosophie können ausgehend von ihrer Selbstbeschreibung auf unbeschränkt viele Themengebiete angewandt werden.

In beiden Fällen kann die Reaktivierung eines traditionellen Selbstverständnisses beobachtet werden: Während Husserl die Wissenschaftlichkeit der Philosophie rehabilitiert, indem er sie als Verbindungsglied zwischen Geistes- und Naturwissenschaften positioniert, rehabilitiert der Wiener Kreis die Philosophie als Instanz, die einen neutralen und unabhängigen Blick auf wissenschaftliche Vorgänge einnehmen kann. Dass die Mitglieder des Wiener Kreises die Philosophie explizit nicht als Fachwissenschaft verstanden wissen möchten, sondern als Methode, die in allen anderen Fachwissenschaften anzuwenden sei, ist bezeichnend. Die Vertreter des Wiener Kreises wenden eine Spezifik der Philosophie, nämlich per Definition keinen eigenen wissenschaftlichen Gegenstand zu besitzen, produktiv um, indem sie die Logik als notwendiges Prinzip aller Wissenschaften rehabilitieren. ${ }^{107}$ Die Logik fungiert im Erneuerungsanspruch des Wiener Kreises auf der einen Seite als sicheres, nicht weiter zu hinterfragendes und ahistorisches Prinzip, um richtige philosophische Probleme von falschen bzw. sinnlosen zu unterscheiden. Auf der anderen Seite nimmt die Logik die Funktion eines neuen philosophischen Gegenstandes ein; als Grundstruktur aller Wissenschaften bietet sie Philosophen und Philosophinnen bis heute eine unendliche Aufgabe, nämlich diese Struktur enträtseln und bestimmen zu können.

107 Die Philosophie als Methode zu definieren, die auf andere Wissenschaften kritisch angewendet werden kann, ist eine Erneuerungsstrategie, die auch bei Husserl und der Kritischen Theorie vorhanden ist. 


\subsection{Eine politisch und wissenschaftlich neutrale Instanz}

In inhaltlicher Hinsicht steht die Neubestimmung der Philosophie des Wiener Kreises in einem diametralen Gegensatz zur Neubestimmung der Philosophie der Kritischen Theorie, wie sie im letzten Kapitel vorliegender Arbeit untersucht wird. Wo die Vertreter des Wiener Kreises die Philosophie in positiver Weise als mathematische Logik definieren, definieren Horkheimer und Adorno die Philosophie in negativer Weise als Kritik. Der Gegensatz beider Neubestimmungen wird im sogenannten Positivismusstreit manifest, der in den 1930er-Jahren mit einem Aufsatz von Horkheimer beginnt ${ }^{108}$ und 1961 in Form einer Auseinandersetzung zwischen Adorno und Karl Popper seinen Höhepunkt erfährt. ${ }^{109}$ Jedoch seien seit dem Ende der 1970er-Jahre - so schreibt Hans-Joachim Dahms - »eine ganze Reihe von Publikationen erschienen [...], die zeigen, dass die Bewegung des logischen Positivismus und insbesondere der Wiener Kreis ähnliche politische Perspektiven verfolgt hat wie die Frankfurter Schule - und dies, wie ich im Vorgriff hinzufügen möchte, häufig praktisch weitaus aktiver als letztere« (ebd.: 15-16). Diese Feststellung ist im Hinblick auf einen Vergleich zwischen den unterschiedlichen Selbstpositionierungsstrategien zentral.Im Folgenden wird deshalb - gewissermaßen als Überleitung zum nächsten Kapitel - auf die politischen Implikationen des philosophischen Erneuerungsanspruches des Wiener Kreises eingegangen.

Wie deutlich geworden ist, nimmt der Wiener Kreis innerhalb der Philosophiegeschichte des 20. Jahrhunderts eine spezielle Rolle ein, da es sich hier um eine Gruppe und nicht um eine Einzelperson handelt. Diese Spezifik widerspiegelt ein zentrales Anliegen der untersuchten Autoren: Philosophie soll nach dem naturwissenschaftlichen Modell als ein Projekt verstanden werden, an dem kollektiv gearbeitet wird. Die Konnotation dieses Modells mit sozialistischer Politik ist naheliegend. Innerhalb der Rezeptionsgeschichte des Wiener Kreises kann diesbezüglich eine Art Paradigmenwechsel beobachtet werden: Bis ungefähr 1970 wurde der Wiener Kreis als ein rein akademischer Zirkel betrachtet und behandelt, der sich mit Logik, Sprachphilosophie und Erkenntnistheorie beschäftigt und eine empiristische, antimetaphysische und

\footnotetext{
108 Es handelt sich um den Aufsatz »Der neueste Angriff auf die Metaphysik« (Horkheimer 1988 [1937]).

109 Der Positivismusstreit sei - zumindest in der Bundesrepublik Deutschland - als Thema »völlig in den Hintergrund getreten«, »allerdings nicht etwa deswegen, weil man zu einer genauen Definition der Problembereiche oder gar zur einvernehmlichen Lösung einzelner Fragen gelangt wäre, sondern mehr weil die begonnene Diskussion im Alltagsbetrieb der Massenuniversitäten ins Stocken geraten und dann gänzlich versandet ist « (Dahms 1994: 15).
} 
formale Philosophie vertreten hat, wobei der soziale und politische Kontext der Gruppe eine untergeordnete Rolle spielte. Das politische Engagement einzelner Mitglieder erschien als eine Beschäftigung, die die eigentliche Philosophie des Wiener Kreises nicht essenziell berührte. Ein Beispiel für diese Art der Rezeption ist das oben zitierte Buch von Ayer Language, Truth and Logic. Der politische und soziale Kontext des Wiener Kreises rückte erst ab den 1970er-Jahren in den Fokus des Interesses. Der allgemeine Ton war: Die Philosophie des Wiener Kreises lässt sich ohne Einbezug ihres kulturellen und politischen Kontextes nicht verstehen. Johann Dvořáks Aufsätze (Dvořák 1985; 1993) sind für diese Art der Rezeption exemplarisch: Laut Dvořák »bemühten sich Vertreter der wissenschaftlichen Weltauffassung des Wiener Kreises um eine nicht-kapitalistische Vergesellschaftung der Wissenschaften« (Dvořák 1985: 133). »Der wahre Radikalismus der wissenschaftlichen Weltauffassung des Wiener Kreises«, schreibt Dvořák, »bestand keineswegs im Physikalismus oder in diversen Parteizugehörigkeiten[,] sondern in der Neubestimmung der gesellschaftlichen Stellung und Aufgaben der Wissenschaft, einer systematischen Verbindung von Wissenschaft, Bildung und Arbeitsleben« (ebd.). Eine weitere frühe Studie dieser Richtung ist das Buch Wittgenstein's Vienna von Allan Janik und Stephen Toulmin. ${ }^{110}$ Janik und Toulmin argumentieren, Wittgensteins Tractatus »should be considered as emerging from a particular cultural milieu « (Janik/Toulmin 1974: 25). In der Rezeptionsgeschichte des Wiener Kreises wird also ein Spannungsverhältnis deutlich: Auf der einen Seite ist er von einer gewissen politischen Aura umgeben, auf der anderen Seite erscheint seine Philosophie als Inbegriff einer formalen, logischen und in diesem Sinne neutralen Philosophie. Innerhalb dieses Spannungsfeldes, so meine Vermutung, liegt die Stärke des Erneuerungsanspruches des Wiener Kreises. Seine Vertreter und Vertreterinnen haben gerade deswegen eine wirkungsvolle philosophische Erneuerung evoziert, weil sie in einer politisch äußerst aufgeladenen Zeit das philosophische Selbstverständnis von Politik entkoppelt haben. Sie verstanden ihre wissenschaftlich-philosophische Arbeit als unpolitisch, aber sie sahen eine soziale Verantwortung in genau diesem Anspruch. Diese These möchte ich zum Abschluss dieses Kapitels und als Überleitung zum nächsten erläutern:

Die Vertreter des Wiener Kreises haben ihre wissenschaftliche Arbeit stets im Kontext gesamtgesellschaftlicher Entwicklungen betrachtet. Beispielsweise schreibt Carnap 1928 im Vorwort zu Der logische Aufbau der Welt:

110 Das Buch von Janik und Toulmin gilt zugleich als ein früher >Klassiker der Wissenschaftsforschung. 
Wir spüren eine innere Verwandtschaft der Haltung, die unserer philosophischen Arbeit zugrundeliegt, mit der geistigen Haltung, die sich gegenwärtig auf ganz anderen Lebensgebieten auswirkt; wir spüren diese Haltung in Strömungen der Kunst, besonders der Architektur, und in den Bewegungen, die sich um eine sinnvolle Gestaltung des menschlichen Lebens bemühen: des persönlichen und gemeinschaftlichen Lebens, der Erziehung, der äußeren Ordnungen im Großen. Hier überall spüren wir dieselbe Grundhaltung, denselben Stil des Denkens und Schaffens. Es ist die Gesinnung, die überall auf Klarheit geht und doch dabei die nie ganz durchschaubare Verflechtung des Lebens anerkennt, die auf Sorgfalt in der Einzelgestaltung geht und zugleich auf Großlinigkeit im Ganzen, auf Verbundenheit der Menschen und zugleich auf freie Entfaltung des Einzelnen. Der Glaube, daß dieser Gesinnung die Zukunft gehört, trägt unsere Arbeit. (Carnap 1928: V-VI)

Carnap stellt eine »innere Verwandtschaft« zwischen der »philosophischen Arbeit« und anderen - sowohl gesellschaftlichen als auch individuellen zeitgenössischen Lebensbereichen fest. ${ }^{111}$ Seine Bemerkung ist zentral; sie verdeutlicht, dass er seine eigene philosophische Arbeit zwar innerhalb gesamtgesellschaftlicher Prozesse verortet, sie aber nicht direkt in den Dienst der Gesellschaft stellt: Im Unterschied zu den Erneuerungsstrategien der Kritischen Theorie, die im nächsten Kapitel untersucht werden, zielt der Wiener Kreis nicht vordergründig darauf, die Philosophie in den Dienst praktisch-gesellschaftlicher Verhältnisse zu stellen. Carnap etwa schreibt in seiner Intellectual Autobiography: »Die meisten von uns, ich eingeschlossen, waren Sozialisten. Aber wir wollten unsere philosophische Arbeit von unseren politischen Zielen getrennt halten. Unserer Ansicht nach waren Logik, auch angewandte Logik, Erkenntnistheorie, Sprachanalyse oder Methodologie der Wissenschaften, wie die Wissenschaften selbst, praktischen Zielen gegenüber neutral, ob moralischen des Einzelnen oder politischen der Gesellschaft« (Carnap 1993 [1963]: 36). ${ }^{112}$ Die Inhalte, Diskussionen, Thesen und Themen des Wiener Kreises waren nicht politisch intendiert, gleichzeitig

111 Auch im »Manifest« wird die enge Beziehung zwischen philosophischen Forderungen und allgemeinen »Lebensfragen« hervorgehoben: »So zeigen zum Beispiel die Bestrebungen zur Neugestaltung der wirtschaftlichen und gesellschaftlichen Verhältnisse, zur Vereinigung der Menschheit, zur Erneuerung der Schule und zur Erziehung einen inneren Zusammenhang mit der wissenschaftlichen Weltauffassung; es zeigt sich, dass diese Bestrebungen von den Mitgliedern des Wiener Kreises bejaht, mit Sympathie betrachtet, von einigen auch tatkräftig gefördert werden « (Neurath/Hahn/Carnap: 304). Gemeint sind hier sozialistische Ideen und Bewegungen dieser Zeit. Das »Manifest« endet mit den Worten: »Die wissenschaftliche Weltauffassung dient dem Leben und das Leben nimmt sie auf« (ebd.: 315).

112 Paul Neurath schreibt dezidiert, dass die meisten Mitglieder Sozialdemokraten waren: »Einige Mitglieder des Kreises [...] waren in der Tat politisch sehr interessiert und zwar 
sahen viele seiner Mitglieder explizit eine soziale und politische Verantwortung in ihrer Arbeit. ${ }^{13}$ Der Erneuerungsanspruch des Wiener Kreises erlangt seine argumentative Stärke gerade aufgrund dieses Zieles, nämlich für die Trennung zwischen Wissenschaft und Gesellschaft bzw. Politik einzustehen. In der Rezeptionsgeschichte des Wiener Kreises verliert die aus Sicht der Gruppe selbst wichtige Trennlinie zwischen gesellschaftspolitischer und philosophischer Arbeit oftmals ihre Kontur: Die Autoren des Kreises werden mithin verallgemeinernd als eine politische Gruppe und ihre Philosophie als politisch intendiert dargestellt. Ein Beispiel ist Michael Friedmans Buch $A$ parting of the ways. Hier erscheint Carnap als ein politischer Denker, der in direkter Opposition zu Heidegger steht: »Carnap and Heidegger are therefore not only at opposite ends of the spectrum philosophically, they are at opposite ends of the spectrum in social and political terms as well« (Friedman 2000: 18). Diese Beobachtung hat durchaus ihre Richtigkeit. Allerdings geht in dieser Art der Rezeption ein zentraler Aspekt verloren, nämlich dass die Wirkkraft der Position des Wiener Kreises gerade darin liegt, gegen die Vereinnahmung der Philosophie durch Politik Stellung zu beziehen. Wie deutlich geworden ist, glauben die Vertreter des Wiener Kreises, mit der Logik ein unabhängiges und neutrales philosophisches Analysemittel gefunden zu haben, wobei dieser Aspekt innerhalb des Kreises - wie oft ausgehend von Neurath - kontrovers diskutiert worden ist. Carnap schreibt etwa in seinen Erinnerungen: »Diese neutralistische Haltung [unserer philosophischen Arbeit] kritisierte Neurath scharf. Wir bestanden aber darauf, daß das Eindringen praktischer und insbesondere politischer Gesichtspunkte die Reinheit der philosophischen Methode trüben sollte« (Carnap 1993 [1963])).

Paul Neurath, Soziologe und Sohn von Otto Neurath, moniert in einem Aufsatz von 1993, dass derWiener Kreis generalisierend als politische Gruppierung wahrgenommen wird und betont das genuin Unpolitische der Gruppe:

als Sozialdemokraten. Ich weiß von keinem der bedeutenderen Mitglieder des Kreises, dass er Kommunist gewesen wäre« (P. Neurath 1993: 637).

113 Die enge Verbindung, die einige Mitglieder des Wiener Kreises zur Volkshochschule eingingen, unterstreicht diesen Punkt. »Vertreter der Wissenschaftlichen Weltauffassung, wie eben Otto Neurath, Edgar Zilsel, Friedrich Waismann, Viktor Kraft, unterrichteten an den Wiener Volkshochschulen. [...] Die Volkshochschule erreichte damals alle Schichten der Bevölkerung. Arbeiter und Angestellte waren in manchen Jahren sogar - verglichen mit ihrem Anteil an der Gesamtbevölkerung - erheblich überrepräsentiert « (Dvořák 1985: 136). Diesbezüglich war Edgar Zilsel das wohl exponierteste Mitglied des Wiener Kreises. »Philosophie ist bei Zilsel an menschliches Handeln und menschlicher Handlungsfähigkeit orientiert, sie soll Zusammenhänge herstellen zwischen den einzelnen Wissenschaftsdisziplinen ebenso wie zwischen Wissenschaft und Alltagsleben« (ebd.: 132). 
Wie aus der einschlägigen Literatur bekannt, waren Entstehung und Thematik des Wiener Kreises von Anfang an und bis zum Schluss völlig apolitisch und hatten mit Politik, weder im parteipolitischen, noch in irgendeinem weiteren gesellschaftspolitischen Sinn überhaupt nichts zu tun. Wie bekannt, entstand der Kreis ursprünglich aus einem privaten Kolloquium im Hause Schlick im Anschluss an seine Philosophischen Vorlesungen an der Universität. [...] Die Thematik, das ist ebenfalls bekannt, konzentrierte sich jahrelang auf eine beinahe satzweise Diskussion von Wittgensteins Tractatus logico-philosophicus. Wer ein noch unpolitischeres Diskussionsthema kennt oder ein solches auch nur erfinden kann, sei hiermit herzlichst eingeladen, das vorzubringen. (Neurath P. 1993: 636-637)

Paul Neurath argumentiert, dass die Philosophie des Wiener Kreises insgesamt nicht politisch motiviert gewesen, sondern es der politisch hoch aufgeladenen Situation zuzurechnen sei, dass sie und insbesondere die Metaphysikkritik des Kreises als linksorientiert wahrgenommen wurden. ${ }^{114}$ Der gesellschaftspolitische Aspekt sei aus einer völlig anderen Richtung gekommen, nämlich aus dem politischen Interesse einiger Mitglieder. Insbesondere die politische Aktivität von Neurath und Hahn habe als Beweis »für die Richtigkeit dieser politischen Einordnung einer im Grunde völlig unpolitischen Gruppe, die regelmäßig zu völlig unpolitischen gelehrten Diskussionen über für die Außenwelt zum Teil recht abstrus und scholastisch klingende Themen zusammenkam«, gegolten (ebd.: 636). Paul Neuraths teilweise polemisch vorgetragene Erinnerungen verweisen aber auf einen zentralen Aspekt im Erneuerungsanspruch des Wiener Kreises: Auf der einen Seite wurde in der Gruppe die Haltung vertreten (und mit einer Identifikation der Philosophie mit den Naturwissenschaften begründet), dass die eigene philosophische Arbeit politisch neutral sei, auf der anderen Seite waren einige Mitglieder des Kreises politisch

114 Paul Neurath stellt fest, dass die Haltung gegen oder für die Metaphysik in der Weimarer Zeit quasi ein politisches Statement bedeutet hat. »Es war aber in jener politisch sehr aktiven Zeit, in der alles, was überhaupt irgendwie zur Diskussion stand, immer auch gleich politisch eingeordnet wurde, so, dass die prononcierten Vertreter einer betont metaphysisch orientierten Philosophie und Wissenschaft zumeist, wenn auch gewiss nicht immer, in politisch konservativen, oftmals auch im aggressiv antidemokratischen Lager zu finden waren, während umgekehrt prononcierte Vertreter einer metaphysikfreien Wissenschaft, soweit sie überhaupt politisch interessiert waren, zumeist, obwohl auch hier gewiss nicht ohne Ausnahmen, demokratischen Gruppierungen oder Parteien angehörten oder solchen zumindest nahestanden oder, wenn sie politisch nicht interessiert waren, doch zumindest eine Art von apolitisch liberalem Standpunkt vertraten, sodass also eine Gruppe wie der Wiener Kreis, die in Wort und Schrift konsistent eine aggressiv anti-metaphysische oder zumindest eine metaphysikfreie Orientierung in Philosophie und Wissenschaft vertrat, sogleich in den Geruch kam, eine linksorientierte Organisation zu sein« (P. Neurath 1993: 635-636). 
aktiv. Im Wiener Kreis und seiner Philosophie, so kann gefolgert werden, wird die Forderung nach einer Unterscheidung zwischen >reiner Wissenschaft und den Bedingungen, unter denen diese Wissenschaft entsteht und besteht, exemplarisch sichtbar. ${ }^{115}$ Schlick macht diese Trennung in einer Klammerbemerkung explizit: »Jede Wissenschaft (sofern wir bei diesem Worte an den Inhalt und nicht an die menschlichen Veranstaltungen zu seiner Gewinnung denken) ist ein System von Erkenntnissen, d.h. von wahren Erfahrungssätzen [...]« (Schlick 1930/31: 7-8). Diese wissenschaftsanalytische Trennung erwies sich im Kontext der 1930er-Jahre in Deutschland - paradoxerweise als politisch aufgeladen. ${ }^{116}$ Ernest Nagel beschreibt in seinen Impressions and Appraisals of Analytic Philosophy in Europe 1936 die Eindrücke, die er bezüglich dieser politischen Dimension während seiner Europareise gesammelt hatte:

It occurred to me that although I was in a city foundering economically, at a time when social reaction was in the saddle, the views presented so persuasively from the Katheder were a potent intellectual explosive. I wondered how much longer such doctrines would be tolerated in Vienna. And I thought I understood at least the partial reason for the vitality and appeal of analytical philosophy. Analytical philosophy is ethically neutral formally [sic!]; its professors do not indoctrinate their students with dogmas as to life, religion, race, or society. But analytical philosophy is the exercise of intelligence in a special field, and if the

115 Diese Unterscheidung wiederum wurde bereits in den 1930er-Jahren operationalisiert. Die Unterscheidung zwischen einem »context of discovery« und einem »context of justification « geht auf Reichenbachs Experience and Prediction von 1938 zurück und ist innerhalb der Philosophy of Science bis heute gültig (Barker/Kitcher 2014: 13). Für die Wissenschaftstheorie - so lautet Reichenbachs Argument verkürzend - sei nur der »context of justification « relevant, also der logische und mathematische Rechtfertigungsvorgang einer neuen wissenschaftlichen Hypothese. Der »context of discovery«, also der psychologische (oder auch historische bzw. soziologische) Entstehungszusammenhang der betreffenden Hypothese, liege nicht im Zuständigkeitsbereich der Wissenschaftstheorie bzw. der Philosophie. Die Frage nach den Entstehungsbedingungen philosophischen Wissens wird anderen Disziplinen zugewiesen. Indem Reichenbach den »context of discovery « aus dem Bereich des philosophisch Relevanten ausschließt, wird umgekehrt argumentativ die Möglichkeit einer Instanz generiert, der wissenschaftliche Neutralität und Objektivität inhärent ist.

116 Der Student Johann Nelböck, der Schlick 1936 erschossen hat, soll beim nachfolgenden Prozess als Motiv angegeben haben, Schlicks Philosophie würde die Jugend verführen (P. Neurath 1993: 636). Zwar wurde Nelböck wegen Mordes und unerlaubten Waffenbesitzes zu zehn Jahren Haft verurteilt, er wurde jedoch nach 18 Monaten aufgrund eines Gnadengesuchs wieder entlassen. »Beim Mord habe es sich um ein Notdelikt gehandelt ein Delikt aus weltanschaulicher und politischer Not. [...] Von diesen weltanschaulichen und politischen Motiven hatte damals [während des ersten Prozesses 1936, MD] nicht gesprochen werden dürfen. Deshalb hatten diese Motive bei dem Prozess nicht berücksichtigt werden können « (Sigmund 2015: 301). 
way of intelligence becomes part of the habitual nature of men, no doctrines and no institutions are safe from critical reappraisals. Because traditional philosophy has so often been practiced as a species of obscurantism, it has become the bete noire of the Wiener Kreis. (Nagel 1936: 9)

Nagel macht die Beobachtung, dass - im Unterschied zur traditionellen metaphysischen Philosophie - die Vertreter und Vertreterinnen der neuen »analytischen« Philosophie ihre Studierenden nicht mit Dogmen bezüglich der Lebensführung, Religion, >Rasse` oder Gesellschaft indoktrinieren würden. Gerade darin liege, so Nagel, die »intellektuelle Sprengkraft« dieser Philosophie. Paradoxerweise ist es also gerade das Formale und Neutrale der analytischen Philosophie, dem Nagel - selbst ein Philosoph der analytischen Richtung - in positiver Weise Sprengkraft zuschreibt. Er fährt wie folgt fort:

I am persuaded that this tension [between speculative and analytical philosophy] exists not merely because traditional speculative philosophy frequently cultivates mystification and conscious irrationalism in matters of strict philosophy, but because it has repercussions upon social theory and practice, as recent events have amply shown. (ebd.)

Nagel führt die »Spannung « zwischen traditioneller spekulativer Philosophie (oder: Metaphysik) und der neuen analytischen Philosophie auf die politische Situation in Europa zurück. Auf der einen Seite bringt er die traditionelle spekulative Philosophie mit den »jüngsten Ereignissen« (vermutlich meint er die Machtübernahme der Nationalsozialisten) in eine Verbindung, auf der anderen Seite beschreibt er die analytische Philosophie wie folgt:

\begin{abstract}
Analytical philosophy has thus a double function: it provides quite green pastures for intellectual analysis, wherein its practitioners can find refuge from a troubled world and cultivate their intellectual games with chess-like indifference to its course; and it is also a keen, shining sword helping to dispel irrational beliefs and to make evident the structure of ideas. It is at once the pastime of a recluse and a terribly serious adventure: it aims to make as clear as possible what it is we really know. (ebd.: 9)
\end{abstract}

Analytische Philosophie biete einen Rückzugsort für abgehobene intellektuelle Spiele und sei gleichzeitig ein Instrument, um irrationalen Überzeugungen ein Ende zu machen. Sie sei ein naiver Zeitvertreib und ein »äußerst ernstes Abenteuer«. Es wird eine Wechselwirkung zwischen beiden Aspekten impliziert: Analytische Philosophie sei gerade deswegen ein »scharfes glänzendes Schwert«, weil sie abgehoben und intellektuell sei. Nagel stellt also fest, dass auch die analytische Philosophie Effekte auf die Praxis hat. Im Gegensatz 
zur traditionellen spekulativen Philosophie werden diese Effekte aber als neutral und in dieser Neutralität als positiv konnotiert; als ein "Schwert«, mit dem gegen irrationale Überzeugungen angekämpft werden könne. Nagel impliziert, dass in der analytischen Philosophie die Möglichkeit einer politisch und ethisch neutralen Instanz enthalten ist.

Der Kampf des Wiener Kreises gegen die Metaphysik erscheint in dieser Perspektive auch als ein Kampf gegen die durch Wertungen und Dogmen geprägte Philosophie der Zeit. Die Ablehnung der Metaphysik ist als Reaktion auf eine Krise zu verstehen, die durch die Verluste wissenschaftlicher und philosophischer Neutralität und Objektivität beschrieben werden kann. Die Bezugnahme auf Alfred Seidel verdeutlicht dies: Seidel hat, wie zu Beginn des Kapitels festgestellt, gewissermaßen zugegeben, dass seine eigene Arbeit von metaphysischen Voraussetzungen durchzogen, d.h. nicht »wertneutral« sei, obwohl es sich nicht um eine metaphysische Fragestellung handele. ${ }^{117}$ Seine Parallelisierung von Metaphysik mit Wertung ist insofern signifikant, weil sie eine für diese Zeit zentrale Herausforderung formuliert: Wie kann die Philosophie ihre wissenschaftliche Objektivität und politische Neutralität noch legitimieren, wenn in den realen gesellschaftspolitischen Verhältnissen sichtbar wird, dass Philosophie mit Wertungen verbunden ist? In der hier eingenommenen Perspektive erscheint die Verfemung der Metaphysik, wie sie der Wiener Kreises vollzieht, nicht nur als »eine Abwehr gegen philosophische Strömungen, die nicht Schritt hielten mit dem damaligen Umschwung der Wissenschaft« (Köhler 1995: 191), sondern gleichzeitig als ein Einstehen für die Neutralität und Objektivität der Wissenschaft und als ein Kampf gegen die Vereinnahmung der Philosophie durch Politik insgesamt. Insofern wird im Erneuerungsanspruch des Wiener Kreises, der durch eine Identifizierung der Philosophie mit den exakten Wissenschaften begründet wird, der Anspruch auf eine Entpolitisierung der Philosophie sichtbar. Die Mitglieder des Wiener Kreises wollen die Philosophie nicht nur von Metaphysik, sondern zugleich von ihrer Verstrickung in die Politik »reinigen «. ${ }^{118}$ In diesem Sinne wiederum ist der Anspruch auf eine formale und neutrale Philosophie, wie sie der Wiener Kreis eingefordert hat, höchst politisch.

An seinem Beispiel, so kann geschlussfolgert werden, wird aufgrund einer Identifikation der Philosophie mit den exakten Wissenschaften die Erneuerung eines traditionellen Selbstverständnisses der Philosophie sichtbar: Philosophie als eine intellektuelle Instanz, die einen neutralen und unabhängigen Blick

117 Siehe S. 11 und 79.

118 Siehe Fn. 95. 
auf politische und wissenschaftliche Vorgänge einnehmen kann. Im nächsten Kapitel wird gewissermaßen das Pendant zum Wiener Kreis thematisiert: Der Erneuerungsanspruch der Kritischen Theorie nimmt seinen Anfang in der Einsicht, dass Philosophie immer mit Politik und Politik immer mit Philosophie verbunden ist. 